\title{
Competitividad y especialización: Un análisis regional de la evolución de la ocupación en España desde una óptica espacial
}

\author{
Mercedes Jiménez García a ${ }^{\text {, José Ruiz Chico }}{ }^{\text {b }}$, Antonio Rafael Peña Sánchez ${ }^{\text {b }}$ \\ a Universidad de Cádiz, Facultad de CC. Económicas y Empresariales., Avda. Duque de Nájera, 8, \\ 11002 Cádiz, España.E-mail: mercedes.jimenezgarcia@uca.es \\ ${ }^{b} \quad$ Universidad de Cádiz, Facultad de CC. Sociales y de la Comunicación, Avda. de la Universidad, s/n, \\ 11405, Jerez de la Frontera (Cádiz), España.E-mail: jose.ruizchico@uca.es, rafael.pena@uca.es
}

\begin{abstract}
RESUMEN
La importancia de la actual crisis económica así como sus efectos sobre el empleo en España en sectores como el de la construcción, motivan el tema de análisis de este artículo cuyo objetivo es conocer la evolución del empleo en las diferentes regiones españolas antes (2001-2006) y durante la actual crisis (2007-2012) en relación con el conjunto de la nación y con las regiones vecinas utilizando, para ello, un análisis shift-share espacialmente modificado. Los resultados muestran el decrecimiento y disminución del peso de la construcción en las diferentes regiones durante la crisis a favor del sector agrícola y servicios. Sólo destaca Castilla-La Mancha como la comunidad en la que el empleo en la construcción crece por encima de la media nacional y tiene mayor peso que en sus regiones vecinas.
\end{abstract}

Palabras clave: Shift-share espacialmente modificado, economía regional, empleo, crisis.

\section{Competitiveness and Specialization: A Regional Analysis of the Evolution of Employment in Spain from a Spatial Perspective}

\begin{abstract}
The importance of the current economic crisis and its effects on employment in Spain in sectors such as building, motivate the subject of analysis in this article, with the aim of studying the evolution of employment in the different Spanish regions before (2001-2006) and during the current crisis (2007-2012) in relation to the whole nation and neighboring regions. We will use, for this purpose, a shift-share analysis spatially modified. The results show the decrease of the weight of building sector in different regions during the crisis, supporting agriculture and services sectors. In general, Castilla-La Mancha highlights as the Community whose building employment has grown faster than the national average, having a greater relevance than its neighboring regions as well.
\end{abstract}

Keywords: Shift-Share Spatially Modified, Regional Economy, Employment, Crisis.

Clasificación JEL: J21, R12

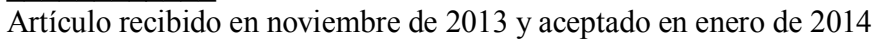

Artículo disponible en versión electrónica en la página www.revista-eea.net, ref. ə-32203 


\section{INTRODUCCIÓN}

La actual situación de crisis económico-financiera por la que está atravesando el mundo en general, es un tema de suma actualidad e interés, tratado en la literatura por diferentes ramas de conocimiento y bajo ópticas y puntos de vista dispares. No obstante, aunque generalizada, esta situación de crisis no está afectando a todos los territorios por igual, midiéndose sus consecuencias con diferente impacto e intensidad en distintos espacios geográficos; de la misma manera que el alcance de sus efectos tampoco es homogéneo en todos los sectores económicos. En España, el sector de la construcción, representa un peso superior en la economía que en otros países europeos. Éste ha sido el principal sector afectado por la pérdida de empleo durante la crisis ${ }^{1}$, a la vez que también se ha caracterizado por ser el sector más dinámico en los años precedentes a la misma (Rodríguez, 2007; García, 2009; Alonso y Furio, 2010; Fernández y Cruz, 2013).

A partir de la recesión de 1993, la economía española inició un nuevo ciclo económico, entrando en una estable y larga etapa de expansión (García, 2012; Fernández y Cruz, 2013), a pesar de la caída de la economía internacional en 1997 motivada, en gran parte, por la crisis de Asia Oriental (Brenner, 2002). El estancamiento económico mundial de finales del siglo pasado y principios del actual afectó en menor medida a España, cuyas tasas de inversión crecieron, sobre todo gracias a la construcción.

Sin embargo, a partir de $2008,{ }^{2}$ la economía española entró en un ciclo contractivo (2008-2009), estancamiento (2010-2011) y recesión (2012) (Navarro, 2008:238; Ortega y Peñalosa, 2012; La Caixa, 2012:47), que se prolonga hasta la actualidad (Álvarez, 2008; Alonso y Furio, 2010; Consejo Económico y Social de España, 2013; Fundación Alternativas, 2013). No obstante, es necesario mencionar que, a partir de 2009, el descenso experimentado por la tasa de crecimiento del PIB español parece haberse suavizado, manteniéndose en un cierto equilibrio, aunque con tendencia a la baja (Alamá y Fuertes, 2009; Sánchez y García, 2011).

El cambio de ciclo provocado por la primera crisis económica española del presente siglo se ha revelado de una gran intensidad y de carácter más cercano a provocar efectos estructurales a nivel del mercado laboral, social, económico, político, etc. que coyunturales (Fernández en VV.AA., 2013:36).

\footnotetext{
${ }^{1}$ Según menciona García (2011:154), “...seis de las ocupaciones que más crecieron durante la expansión y ocho de las que más se redujeron durante la recesión estaban asociadas directa o indirectamente al sector de la construcción..." -de un listado de diez-.

2 Algunos autores, como García (2011:150) y Álvarez et al. (2013), sitúan el punto de inflexión en el ciclo económico a finales de 2007.
} 
La observación de la evolución de estos ciclos económicos en España lleva a la selección de dos periodos de análisis: por una parte, el comprendido entre 2001 y 2006, periodo de seis años precedentes a la llegada de la actual crisis a España, en los que se observa una tendencia global de cierta estabilidad en el comportamiento de unas tasas de crecimiento altas del PIB español (en torno al $4,2 \%$ ) tras el estancamiento económico mundial de finales de los noventa. Por otra parte, otros seis años correspondientes al actual periodo de crisis (20072012), caracterizado por un fortísimo descenso del crecimiento del PIB, experimentando incluso valores negativos en 2012.

Con este trabajo se pretende realizar una aportación adicional al estudio de las implicaciones de la crisis actual en el empleo de los diferentes sectores de actividad en las Comunidades Autónomas (CCAA) españolas, centrándose, fundamentalmente, en el sector de la construcción. ${ }^{3}$ Siendo conscientes de que es necesario una perspectiva largo placista para poder analizar en profundidad los efectos y consecuencias de la actual crisis sobre la variable empleo y otras variables macroeconómicas, con en este trabajo se pretende ofrecer una primera aproximación a las mismas. Para ello se establece una comparativa de la evolución del empleo antes (2001-2006) y durante la crisis económica (2007-2012) en las diferentes regiones en relación con el comportamiento en el conjunto de la nación, incluyendo como una variable a considerar en el estudio la influencia de las regiones españolas vecinas y desagregando el análisis por sectores de actividad (agricultura, industria, construcción y servicios).

Para alcanzar el objetivo planteado, se utiliza el análisis shift-share espacialmente modificado (frente al análisis shift-share del empleo, utilizado, tradicionalmente, en función de las diferentes regiones y sectores de actividad Ezcurra y Rapún (2006); Fotopoulos (2007); Cörvers y Meriküll (2007), etc.-), ya que se considera la herramienta más adecuada al permitir la identificación de las regiones afectadas por un cambio en su estructura de empleo antes y durante la crisis, y la descomposición del mismo en función de su especialización, dinamismo y ventaja/desventaja competitiva en el sector de la construcción, fundamentalmente.

El trabajo se estructura de la siguiente manera: en el segundo apartado se describen los antecedentes y la situación actual del contexto de análisis geográfico y temporal, con especial referencia al empleo en el sector de la construcción; en el tercer apartado se detalla la metodología empleada en el análisis de la variable empleo, es decir, el shift-share espacialmente modificado; el cuarto apartado se centra en la evolución del empleo regional en los dos periodos de estudio, antes y durante la crisis actual; y en el epígrafe final se reúnen las principales ideas y conclusiones del análisis efectuado.

\footnotetext{
${ }^{3}$ Véanse los estudios previos relativos a la situación del empleo durante la crisis de Navarro et al. (2011); García (2012) y Sanromà (2012), entre otros.
} 


\section{ANTECEDENTES Y SITUACIÓN ACTUAL DEL EMPLEO EN ESPAÑA, ESPECIAL REFERENCIA AL SECTOR DE LA CONSTRUCCIÓN}

El mercado laboral español es especialmente sensible a las etapas de crecimiento-decrecimiento de los ciclos económicos (Recio, 2013:54), hecho que se manifestó en la etapa expansiva precedente a 2007 en la que el aumento del empleo en España superó ampliamente la media europea (en el periodo 19992007), así como durante la actual situación de crisis, situándose España como el primer país de la Unión Europea (UE) en lo que a destrucción de empleo se refiere (Ayala et al., 2009; Medina et al., 2010:38; García, 2011:151). ${ }^{4}$

Figura 1

Evolución de la tasa de paro en España y en la UE-28

(en porcentaje)



Fuente: Elaboración propia a partir de Eurostat.

En la Figura 1 se recoge la evolución de la tasa de paro en España en relación con la de la UE-28 durante el periodo objeto de análisis (2001-2012), observándose una tendencia ligeramente decreciente y paralela en las mismas durante los cuatro años previos a la llegada de la actual crisis económica. A partir de ahí, desde 2007, ambas han seguido tendencias similares pero de forma muy distanciada. La tasa de desempleo española experimentó un importante aumento en su tasa de crecimiento, doblando en 2009 a la tasa de paro europea (18\% frente al $9 \%$ de la población activa, respectivamente) y superando el doble de ésta desde entonces. En España, la tasa de paro ha experimentado un crecimiento mínimo de 1,58 puntos porcentuales en el periodo 2010-2011, y un má-

\footnotetext{
${ }^{4}$ Es necesario precisar, tal como indican Conde et al. (2011:157), que a pesar de la importante destrucción de empleo en España, motivado, fundamentalmente, por las altas tasas de temporalidad en el empleo así como por actividades productivas en crisis muy intensivas en trabajo (Muñoz y Antón, 2011), la caída en su actividad económica ha sido menor que en la mayoría de los países de la Unión.
} 
ximo de 6,67 puntos porcentuales en 2008-2009. En otras palabras, desde el inicio de la actual crisis mundial, a mediados de 2007, la tasa de desempleo española ha aumentado del $8 \%$ al 25\% en 2012 (Álvarez et al., 2013), muy alejada de las cifras europeas, que han pasado del 7\% al 10,5\% para los mismos años.

Tal y como se mencionó en el apartado anterior, en referencia al ciclo económico, la evolución de las cifras de la Figura 1 responden a un periodo de expansión del mercado laboral español (1998-2007), procedente, fundamentalmente, del incremento tanto de los flujos migratorios como de la tasa de actividad femenina, seguido de una etapa de recesión (a partir de 2008) (Bentolila et al., 2010:5). Esta mencionada recesión conlleva aspectos más allá de la reducción de la tasa de ocupación y el aumento del paro, abarcando también problemas de pérdida de bienestar social, riesgo de exclusión y pobreza, precariedad en la contratación o aumento del paro de larga duración, entre otros (Gradín et al., 2012; Álvarez et al., 2013).

Sin embargo, la situación de crisis no ha afectado por igual al empleo en los diferentes sectores económicos, debido a la dependencia de la economía española del sector inmobiliario y de la construcción en los años precedentes a la crisis $^{5}$ (Pérez, 2006; Fernández y García, 2008:59; García, 2011; Consejo Económico y Social de España, 2013; Recio, 2013:68). Este último, se ha configurado como el sector más dinámico antes de la llegada de la crisis a España, tanto por su contribución al PIB -este sector aumentó su valor añadido bruto en un 43\% entre 2000 y 2007 (Fernández y Cruz, 2007:8)-, como por el efecto multiplicador que genera por su relación con el resto de actividades económicas y, sobre todo, por el empleo directo e intensivo de mano de obra (Alonso y Furio, 2010; Bentolila et al., 2010:7).

En la Figura 2 se representa la tasa de crecimiento del número de ocupados en España en cada sector de actividad -la tasa de ocupación se considera, junto con la productividad, un factor determinante de la evolución de la renta per cápita y de la capacidad del Estado para atender el bienestar (Peña, 2011; Álvarez et al., 2013; Peña y Jiménez, 2013)-. La observación de la misma refuerza lo comentado en el párrafo anterior. Desde comienzos de siglo, y hasta la llegada de la crisis, los sectores agrícola e industrial, presentaban tasas de crecimiento negativas anuales en su número de ocupados (fundamentalmente Galicia, Castilla-La Mancha, La Rioja y Murcia, en el sector agrícola y Cataluña, Aragón y País Vasco en el sector industrial). Comportamiento éste opuesto al experimentado en los sectores de la construcción y servicios, donde se concentró el aumento de la ocupación. El sector de la construcción mostró una tasa de

\footnotetext{
5 “...España partía de un peso de la construcción en el empleo total (10,7\%) que era el más elevado junto a Portugal en 1999 y la evolución posterior de la ocupación le llevó a mantener dicha posición junto a Irlanda en 2007, muy lejos de la media europea" (García, 2011:151).
} 
crecimiento en su número de ocupados positiva de 2001 a 2006 en doce de las diecisiete CCAA, mientras que el sector servicios experimentó este comportamiento en todas las regiones españolas, para el mismo periodo.

Figura 2

Tasa de crecimiento de los ocupados en España por sector económico (en porcentaje)

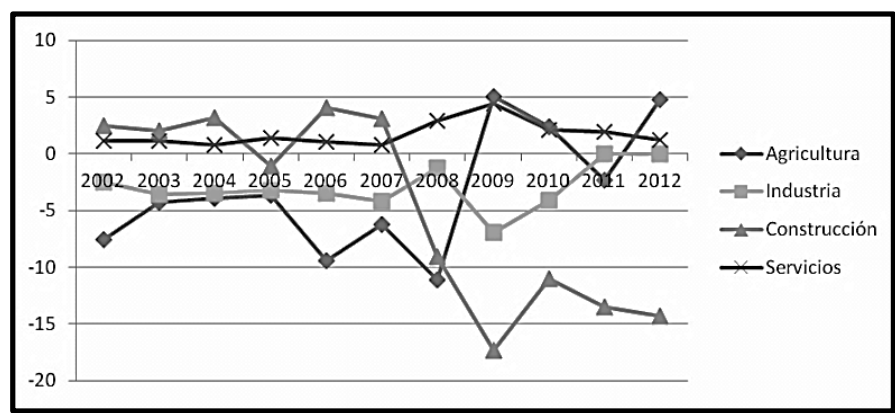

Fuente: Elaboración propia a partir de INE.

No obstante, a partir de 2007, esta tendencia se invierte. La mayor parte de la caída en el empleo se concentra en el sector de la construcción, hasta 2008 compensada parcialmente por el aumento de empleados en los servicios y asumiendo pequeñas pérdidas en los otros sectores. Pero la caída más intensa en la ocupación se produjo entre el último trimestre de 2008 y el primero de 2009 cuando a la destrucción de empleos en la construcción se une la industria (con una pérdida de 650 mil ocupados) (García, 2012:170).

A nivel regional, el sector de la construcción ha sido el único sector en España que ha experimentado tasas de crecimiento en sus ocupados negativas para todas las CCAA durante el periodo 2007-2012, además de los valores más elevados de las mismas, en relación al conjunto de la actividad económica. Las tasas de decrecimiento del desempleo en el sector han sido muy superiores a las de crecimiento del periodo anterior a la crisis, y han alcanzado su mínimo en 2009. Las comunidades más afectadas fueron las del sur de España: Murcia, Andalucía, Canarias y Castilla-La Mancha.

Paralelamente, en la agricultura la tasa de crecimiento del empleo comienza a tomar valores positivos (en comunidades como Murcia, Extremadura o Valencia, entre otras) y en la industria se observa también una ligera recuperación, con tasas menos negativas que antes de la crisis e incluso, en los últimos años, igual a cero (País Vasco y Cataluña han sido las comunidades que más afectadas se han visto por el decrecimiento del empleo industrial).

El sector servicios, por su parte, alcanzó su máxima tasa de crecimiento en 2009, al igual que la agricultura (coincidiendo con la principal caída en la cons- 
trucción) -muchas de las personas empleadas en el sector de la construcción, normalmente con baja cualificación profesional, vuelven o se re-emplean en sectores como el agrícola o la hostelería, dentro del sector servicios, para hacer frente a su situación de desempleo-, para, a partir de ahí, continuar con una tendencia decreciente llegando, en 2012, a ostentar cifras de crecimiento similares en sus ocupados que antes de la crisis, en 2005 y 2006. Las comunidades que experimentaron un mayor crecimiento de este sector fueron Canarias, Andalucía y Valencia, principalmente por la importancia del turismo en sus economías, mientras que en La Rioja y Extremadura fue donde menor aumento se produjo en el número de ocupados en servicios durante la crisis.

Entre los colectivos más afectados por el desempleo, se pueden mencionar a la población joven y poco cualificada y a los inmigrantes, hecho que aumenta su riesgo de exclusión social (Álvarez et al., 2013). En cuanto a estos últimos, es en la construcción en el sector que más se emplean (en enero de 2007 los extranjeros representaban el 19,7\% del total de ocupados en ese sector), concentrándose, fundamentalmente, en los puestos más bajos (como peones) (Pajares, 2007).

Sin embargo, el mercado laboral español no sólo se ve afectado por las altas tasas de desempleo, muy por encima de la media de la UE, como ya se ha comentado, sino también por la elevada temporalidad del mismo y su escasa movilidad (Cebrián y Moreno, 2012; Recio, 2013:51). Además, durante la crisis se ha observado un cambio en su estructura, con un incremento del peso relativo de la contratación a tiempo parcial, lo que supone menores ingresos en forma de salario e insatisfacción laboral de los ocupados, al no corresponder, en la mayoría de los casos, a un cambio de preferencia de los trabajadores en cuanto al tipo de jornada laboral sino a un intento de flexibilización del mercado para adaptarse a la crisis (Moral et al., 2012; Álvarez et al., 2013).

Tanto el aumento de representatividad de la jornada a tiempo parcial como la destrucción de empleo observada en el mercado laboral español desde finales de 2007 tienen una incidencia directa sobre dos macromagnitudes fundamentales para el sostenimiento de la economía: el consumo y el ahorro. A su vez, el Estado también se ve afectado por un incremento del déficit público al reducir los ingresos vía cotizaciones a la Seguridad Social y aumentar el gasto público vía subsidios por desempleo (López, 2013:42).

\section{METODOLOGÍA: DEPENDENCIA ESPACIAL Y ANÁLISIS SHIFT-SHARE}

El análisis shift-share es una de las herramientas más empleadas en economía regional para medir los componentes del crecimiento económico de determinadas áreas geográficas (municipios, comarcas, regiones, países, etc.). El modelo original de Dunn se basa en la descomposición del crecimiento de una 
magnitud económica entre dos momentos del tiempo en tres efectos: nacional, sectorial o estructural y competitivo o regional (Dunn, 1960).

No obstante, al planteamiento clásico del shift-share se le han realizado diversas críticas entre las que se pueden mencionar, fundamentalmente, la ausencia de contenido teórico, los problemas de agregación, la subestimación de la influencia de la estructura industrial, la inestabilidad temporal del efecto competitivo, la interdependencia de los efectos sectorial/regional o la imposibilidad de contrastar la validez estadística de los efectos detectados, entre otras (Esteban-Marquillas, 1972; Richardson, 1978; Dawson, 1982; Arcelus, 1984; Dinc et al., 1998; Garrido, 2002; Wadley y Smith, 2003; Ramajo y Márquez, 2008).

En respuesta a estas críticas, el planteamiento clásico del shift-share se ha visto sometido a diversas transformaciones, revisiones y extensiones. En lo que respecta a la dotación al modelo de un marco teórico, se pueden destacar los trabajos de Chalmers y Beckhelm (1976), Sakashita (1973), Casler (1989) o Graham y Spence (1998). En cuanto a la segunda crítica realizada, autores como Fothergill y Gudgin (1979) y Casler (1989), mencionan que la desagregación no llega a suponer grandes problemas de sesgo, y sobre la tercera crítica, si se analiza un corto periodo de tiempo, el problema planteado no se considera tal, ya que es poco probable que la estructura industrial experimente cambios. Además, como otra alternativa, Fuchs (1959) propone realizar el promedio entre el primer y último año. Gerking y Barrington (1981) demostraron, a través de un estudio empírico, que la inestabilidad del componente competitivo no invalida el análisis shift-share como método de predicción. Y, finalmente, en respuesta a las dos últimas críticas expuestas, Esteban-Marquillas (1972) propone una reformulación del modelo y autores como Theil y Gosh (1980), Berzeg y Koran (1984) y Knudsen y Barf (1991) elaboran trabajos en los que se dota al análisis de una estructura probabilística.

Por lo tanto, las críticas recibidas al análisis shift-share encuentran respuesta y/o solución en una amplia variedad de literatura por lo que, se sigue considerando una herramienta válida en el análisis económico regional, ya sea con fines predictivos, de evaluación de políticas, de planificación estratégica, etc. (Ramajo y Márquez, 2008).

En la actualidad, la creciente facilidad de acceso a datos de mayor desagregación, el interés por la economía regional y el desarrollo de la econometría espacial, plantean la necesidad de incorporar la influencia del espacio en el análisis shift-share (Mayor y López, 2005; Ramajo y Márquez, 2008), ya que éste considera a las unidades geográficas como independientes entre sí. Además, autores como Isard (1960), Hewings (1976) y Mayor y López (2005), entre otros, reconocen la existencia de interacciones espaciales entre territorios vecinos y la primera ley de la geografía de Tobler o también denominado principio de autocorrelación espacial refiere que "todas las cosas están relacionadas 
entre sí, pero las cosas más próximas en el espacio tienen una relación mayor que las distantes" (Cliff y Ord, 1973; Tobler, 1979).

Con la finalidad de incorporar el componente espacial al análisis shift-share, surgen modelos que introducen las matrices de pesos espaciales para contabilizar el papel que juegan las regiones vecinas en el crecimiento de un territorio, tales como los propuestos por Nazara y Hewings (2004), por Mayor y López (2005) o por Ramajo y Márquez (2008). ${ }^{6}$

En esta investigación se ha empleado el shift-share espacialmente modificado propuesto por Mayor y López (2005:14), en el que se combina el enfoque de shift-share espacial de Nazara y Hewings (2004) con la consideración de la influencia espacial en la variable de estudio, en función de la estructura de la matriz de pesos espaciales. Esta metodología ha sido aceptada por diversos autores y empleada tanto en sus referencias como en sus aplicaciones a casos prácticos (véanse, por ejemplo, Arias y Sánchez, 2010:15; Yánez y Acevedo, 2010: 92; Esteban et al., 2010; Arias y Sánchez, 2011:406).

Por lo tanto, la expresión del shift-share espacialmente modificado para el empleo sería la siguiente:

$$
E_{i j}^{\prime}-E_{i j}=\Delta E_{i j}=E_{i j} r+E_{i j}\left(r_{i}-r\right)+E_{i j}^{v^{* *}}\left(r_{i j}-r_{i}\right)+\left(E_{i j}-E_{i j}^{v^{* *}}\right)\left(r_{i j}-r_{i}\right)
$$

Donde,

$E_{i j}^{\prime}$ : valor de la variable empleo en el periodo final, correspondiente al sector $i(\mathrm{i}=1, \ldots, \mathrm{S})$ en el ámbito espacial $j(\mathrm{j}=1, \ldots, \mathrm{R})$.

$E_{i j}$ : valor del empleo en el periodo inicial, correspondiente al sector $i$ $(\mathrm{i}=1, \ldots, \mathrm{S})$ en el ámbito espacial $j(\mathrm{j}=1, \ldots, \mathrm{R})$.

$$
\begin{aligned}
r=\frac{\sum_{i=1}^{S} \sum_{j=1}^{R}\left(E_{i j}^{\prime}-E_{i j}\right)}{\sum_{i=1}^{S} \sum_{j=1}^{R} E_{i j}} \quad r_{i} & =\frac{\sum_{j=1}^{R}\left(E_{i j}^{\prime}-E_{i j}\right)}{\sum_{j=1}^{R} E_{i j}} \quad r_{i j}=\frac{E_{i j}^{\prime}-E_{i j}}{E_{i j}} \\
E_{i j}^{v^{* *}} & =E_{j} \frac{E_{i}^{v^{*}}}{E^{v^{*}}}
\end{aligned}
$$

siendo

$$
\frac{E_{i}^{v^{*}}}{E^{v^{*}}}=\frac{\sum_{j=1}^{R} E_{i j}^{v^{*}}}{\sum_{i=1}^{S} \sum_{j=1}^{R} E_{i j}^{v^{*}}}
$$

\footnotetext{
${ }^{6}$ Otra opción que se puede encontrar en la literatura para incluir el efecto espacial en el análisis shift-share, sin utilizar la matriz de pesos, es emplear el concepto de empleo homotético (Esteban-Marquillas, 1972) referenciado a las regiones vecinas y no al conjunto del país (Mayor y López, 2005: 13). No obstante, la metodología utilizada en este artículo se considera de mayor elaboración y no incurre en algunas de las debilidades detectadas en el modelo de EstebanMarquillas (Loveridge y Selting, 1998; Mayor y López, 2005: 13).
} 
$\mathrm{y}$

$$
E_{i j}^{v^{*}}=\sum_{k \in V} w_{j k} E_{i k}
$$

Entendiéndose por $V$ al conjunto de las regiones vecinas de $j, E_{i j}^{v^{*}}$ la magnitud empleo en función de la influencia de los valores vecinos correspondiente al sector $i(\mathrm{i}=1, \ldots, \mathrm{S})$ en el ámbito espacial $j(\mathrm{j}=1, \ldots, \mathrm{R})$ y $w_{j k}$ el elemento correspondiente de la matriz de pesos espaciales estandarizada por filas, tal y como se definirá posteriormente.

Los dos primeros sumandos de la expresión (1) coinciden con los efectos del análisis shift-share clásico: efecto nacional $\left(E_{i j} r\right)$ y efecto sectorial o estructural $\left(E_{i j}\left(r_{i}-r\right)\right)$. Donde, el efecto nacional refleja el crecimiento que tendría el sector $i$ en la región $j$ si evolucionara de acuerdo con la economía nacional, es decir, representa el efecto de arrastre de la economía nacional; y el efecto sectorial detecta si un sector crece por encima o por debajo de la media nacional (signo positivo y negativo, respectivamente), identificando, de esta manera, los sectores más dinámicos (Delgado et al., 1997:13; Duarte et al., 2000).

Sin embargo, el efecto regional o competitivo clásico $\left(E_{i j}\left(r_{i j}-r_{i}\right)\right)$, que relaciona el crecimiento de un sector en una región con la media sectorial de la nación, se ve modificado al introducir la influencia de los territorios vecinos, dando lugar a dos efectos diferentes: el efecto competitivo neto $E_{i j}^{v^{* *}}\left(r_{i j}-r_{i}\right)$ y el efecto locacional espacialmente modificado $\left(E_{i j}-E_{i j}^{v^{* *}}\right)\left(r_{i j}-r_{i}\right)$.

El primero -al que de ahora en adelante se le denominará E.C.N.E.-, expresa la ventaja o desventaja competitiva de la región en el sector $i$ respecto a ese sector en las regiones vecinas, de forma que un signo positivo de este efecto indicará que el sector, en esa región, crece más rápidamente que en las regiones limítrofes.

El segundo -al que de ahora en adelante se le denominará E.L.E.- mide el grado de especialización sectorial de cada región, identificando si se encuentra especializada en las ramas en las que tiene ventaja competitiva respecto a las regiones vecinas (Arias y Sánchez, 2010). El signo de este efecto depende del producto de dos factores. El primero de ellos $\left(E_{i j}-E_{i j}^{v^{* *}}\right)$ compara la magnitud de la variable estudiada, en este caso, el empleo, de un sector en una región con la que se obtendría si ese sector tuviera el mismo peso en la economía regional que en la del conjunto de las regiones vecinas, refleja por lo tanto el peso o la participación relativa del sector en la economía regional en relación con las regiones colindantes. Mientras que el segundo factor $\left(r_{i j}-r_{i}\right)$ indica si el sector crece más a nivel regional que a nivel nacional. Por lo tanto, un signo de E.L.E. positivo se puede deber a que ambos factores sean positivos o negativos, algo que se conoce al observar el signo de E.C.N.E. (Duarte et al., 2000). 
Además, del análisis shift-share también se puede obtener el Efecto Total Neto (E.T.N.) como sumatorio de los efectos sectorial y regional total, que representa, para cada región y sector, el crecimiento diferencial con respecto a la media nacional (Delgado et al., 1997:13).

No obstante, antes de someter el territorio objeto de estudio a un análisis shift-share espacialmente modificado, ${ }^{7}$ es necesario detectar la presencia de autocorrelación espacial, es decir, analizar si la variable estudiada (en nuestro caso, el empleo) en una subregión $j$ es independiente del valor de esa misma variable en las subregiones vecinas (Arias y Sánchez, 2010:14). Para ello, se pueden emplear diversos tests como la C de Geary, la G(d) de Getis y Ord, LMERR o la I de Moran, entre otros (Moreno y Vayá, 2000:35 y ss.).

En este caso, se ha utilizado este último, al ser el que, de los contrastes adhoc, más garantías ofrece (Mur, 1992:289). La expresión matemática del índice de Moran es la siguiente:

$$
I=\frac{n}{S_{0}} \frac{\sum_{j=1}^{n} \sum_{k=1}^{n} w_{j k} z_{j} z_{k}}{\sum_{j=1}^{n} z_{j}^{2}} ; j \neq k
$$

donde, $z_{j}=x_{j}-\bar{x} \quad$ y $S_{0}=\sum_{j=1}^{n} \sum_{k=1}^{n} w_{j k}$

Siendo $n$ el número de casos, $x_{j}$ el valor de la variable en un lugar determinado, $x_{k}$ el valor de la variable en otro lugar diferente, $\bar{x}$ la media de la variable y $w_{j k}$ el peso aplicado a la comparación entre la localización $j$ y la localización $k$.

Por lo tanto, el cálculo de la I de Moran requiere la construcción previa de la matriz de pesos espaciales $W$, siendo ésta una matriz cuadrada no estocástica cuyos elementos $w_{j k}$ miden la interdependencia entre unidades territoriales a través de criterios de contigüidad en función de una distancia basada ya sea en las relaciones comerciales entre las mismas, la longitud de la frontera común, la renta per cápita, los niveles de empleo, etc.

$$
W=\left[\begin{array}{cccc}
0 & w_{12} & \cdot & w_{1 n} \\
w_{21} & 0 & \cdot & w_{2 n} \\
\cdot & \cdot & \cdot & \cdot \\
w_{n 1} & w_{n 2} & \cdot & 0
\end{array}\right]
$$

En el caso concreto que nos ocupa, se ha empleado un indicador de la distancia económica (Case et al., 1993) para construir la matriz de pesos. Según Ghemawat (2001:5), la distancia económica incluye las diferencias en las rentas

\footnotetext{
${ }^{7}$ A pesar de que el análisis shift-share espacial se vaya a efectuar para las 17 CCAA españolas, las comunidades de Baleares y Canarias, al ser islas, no cuentan con regiones vecinas, por lo que para estas dos regiones, el análisis shift-share no se ve modificado por el componente espacial.
} 
de los consumidores, y las diferencias en el coste y calidad de los recursos naturales, financieros, humanos, infraestructuras, productos intermedios, y conocimientos. En este sentido, atendiendo a este concepto y siguiendo el trabajo de Cuervo (2008:100), se utilizan los componentes del Indice de Desarrollo Humano (IDH) de Naciones Unidas como indicador adecuado para medir esta distancia. Se ha empleado el nuevo método para calcular el IDH, surgido a partir del informe de la ONU del año $2010,{ }^{8}$ como media geométrica de los índices normalizados de vida, educación e ingresos $\left(\mathrm{IDH}=\mathrm{I}_{\mathrm{Vida}}{ }^{1 / 3}\right.$. $\mathrm{I}_{\text {Educación }}{ }^{1 / 3}$. $\mathrm{I}_{\text {Ingreso }}{ }^{1 / 3}$ ).

De esta manera, se elabora el IDH para las diecisiete CCAA españolas para los dos periodos analizados, ${ }^{9}$ a partir de los cuales se obtienen los diferentes elementos $w_{j k}$ de la matriz de pesos espaciales. Cada elemento de la matriz se define como $w_{j k}=\frac{1}{\left|x_{j}-x_{k}\right|}$, entendiéndose $x$ como el IDH, $j$ y $k$ como unidades espaciales diferentes -cada una de las Comunidades Autónomas- y $w$ como la intensidad de interdependencia entre ellas -en este caso, en función del criterio de distancia económica marcado por el IDH-.

La matriz de pesos se ha estandarizado, acotando los pesos entre 0 y 1 , de forma que en la diagonal principal todos sus elementos son igual a cero $\left(w_{j k}=0\right)$; los componentes de cada fila suman uno y el valor de una variable en un territorio es igual a su media ponderada en las zonas vecinas $[\check{X}=W X]$.

Las fuentes estadísticas consultadas para la realización de este trabajo han sido las bases de datos del Instituto Nacional de Estadística (INE), del Ministerio de Educación, Cultura y Deporte y de la Organización de Naciones Unidas (ONU).

\section{EVOLUCIÓN DEL EMPLEO REGIONAL ANTES Y DURANTE LA CRISIS ECONÓMICA}

Tal y como se comentó anteriormente, en el epígrafe metodológico, para detectar la presencia de autocorrelación espacial se ha realizado el Test de Moran para el IDH en los dos periodos objeto de estudio.

Se muestra, a partir de la Tabla 1, la presencia de autocorrelación espacial positiva para ambos periodos ( $\mathrm{I}>0$ y se rechaza la Hipótesis nula a un nivel de significación del $5 \%$ ). Estos resultados sugieren que comunidades con un alto/bajo empleo, se encuentran próximas a otras con niveles relativamente al-

\footnotetext{
${ }^{8}$ Véase Klugman et al. (2011) y ONU (2013).

${ }^{9}$ Los datos referentes a la variable educación se han obtenido del S.G. de Estadística y Estudios del Ministerio de Educación, Cultura y Deporte, mientras que los relativos a las otras dos variables se obtienen a partir del Instituto Nacional de Estadística. Para las variables de esperanza de vida y educación sólo se han dispuesto de datos hasta 2011.
} 
tos/bajos del mismo también (Ordóñez et al., 2011). Esta relación se observa también en el segundo periodo estudiado, correspondiente a la crisis.

Tabla 1

Test de autocorrelación espacial

\begin{tabular}{|c|c|c|c|}
\cline { 2 - 4 } \multicolumn{1}{c|}{} & I de Moran & Z(i) & p-valor \\
\hline $\mathbf{2 0 0 1 - 2 0 0 6}$ & 0,2680 & 1,7155 & 0,0427 \\
\hline $\mathbf{2 0 0 7 - 2 0 1 2}$ & 0,2869 & 5,2908 & $\approx 0$ \\
\hline
\end{tabular}

Fuente: Elaboración propia.

Para analizar el empleo se ha tomado la variable, proporcionada por el INE, ocupados por sector económico y Comunidad Autónoma, para el periodo 20012012.

Tabla 2

Efecto Nacional (E.N.) y Efecto Total Neto (E.T.N.) de los ocupados, 2001-2006

\begin{tabular}{|l|c|c|c|c|}
\cline { 2 - 5 } \multicolumn{1}{c|}{} & \multicolumn{4}{c|}{ E.N.+E.T.N. } \\
\cline { 2 - 5 } \multicolumn{1}{c|}{} & AGRICULTURA & INDUSTRIA & CONSTRUCCIÓN & SERVICIOS \\
\hline Andalucía & $-22,33$ & 22,62 & 167,58 & 529,68 \\
\hline Aragón & 0,67 & $-5,15$ & 13,13 & 79,85 \\
\hline Asturias & $-2,58$ & 3,93 & 7,48 & 50,28 \\
\hline Baleares & 1,05 & 3,98 & 7,45 & 89,50 \\
\hline Canarias & $-7,73$ & 13,05 & 16,93 & 145,55 \\
\hline Cantabria & $-4,88$ & 1,50 & 9,65 & 36,28 \\
\hline Castilla y León & $-6,10$ & 8,33 & 26,38 & 114,03 \\
\hline Castilla - La Mancha & $-12,60$ & 32,45 & 37,13 & 108,03 \\
\hline Cataluña & 14,28 & $-23,98$ & 115,75 & 486,88 \\
\hline Comunidad Valenciana & $-17,33$ & 45,50 & 80,87 & 320,98 \\
\hline Extremadura & $-1,00$ & 6,13 & 6,70 & 43,33 \\
\hline Galicia & $-45,43$ & 14,65 & 14,75 & 149,30 \\
\hline Madrid & 11,55 & $-19,55$ & 101,03 & 517,43 \\
\hline Murcia & $-3,20$ & 10,78 & 44,13 & 84,30 \\
\hline Navarra & $-2,75$ & 2,27 & 8,50 & 30,60 \\
\hline País Vasco & $-0,17$ & $-6,63$ & 4,33 & 110,60 \\
\hline Rioja (La) & $-2,13$ & 6,43 & 5,23 & 22,20 \\
\hline
\end{tabular}

Fuente: Elaboración propia.

El componente estructural del paro en la economía española (de la Dehesa, 2003; Izquierdo y Regil, 2006) se refleja incluso durante un ciclo económico favorable, ya que en el periodo 2001-2006 sólo Baleares experimentó un crecimiento del empleo simultáneo en los cuatro sectores analizados, siendo el sector agrícola el más afectado en este sentido, ya que doce de las diecisiete CCAA, 
ven aumentado el empleo en industria, construcción y servicios, pero no en agricultura. Sin embargo, a pesar de esto, en la mayoría de comunidades, tres de los cuatro sectores muestran un crecimiento en el empleo en el periodo analizado y ninguna presenta decrecimiento en todos ellos (Tabla 2).

Un comportamiento totalmente diferente se observa en el periodo siguiente de crisis, 2007-2012, (Tabla 3) en el que quince de las diecisiete CCAA muestran una tendencia recesiva del empleo en todos los sectores, a excepción de Baleares y Murcia que sólo alcanzan valores positivos en el sector servicios y agrícola, respectivamente (IVIE, 2011).

\section{Tabla 3}

Efecto Nacional (E.N.) y Efecto Total Neto (E.T.N.) de los ocupados, 2007-2012

\begin{tabular}{|c|c|c|c|c|}
\hline & \multicolumn{4}{|c|}{ E.N.+E.T.N. } \\
\hline & AGRICULTURA & INDUSTRIA & CONSTRUCCIÓN & SERVICIOS \\
\hline Andalucía & $-45,55$ & $-89,88$ & $-334,75$ & $-121,23$ \\
\hline Aragón & $-4,33$ & $-30,15$ & $-33,68$ & $-8,90$ \\
\hline Asturias & $-3,73$ & $-18,80$ & $-20,38$ & $-14,25$ \\
\hline Baleares & $-6,13$ & $-15,08$ & $-35,90$ & 13,83 \\
\hline Canarias & $-12,03$ & $-14,60$ & $-94,60$ & $-43,28$ \\
\hline Cantabria & $-1,93$ & $-8,33$ & $-18,05$ & $-1,07$ \\
\hline Castilla y León & $-10,33$ & $-38,35$ & $-58,55$ & $-22,63$ \\
\hline Castilla - La Mancha & $-8,53$ & $-27,00$ & $-81,15$ & $-15,45$ \\
\hline Cataluña & $-22,45$ & $-224,10$ & $-252,10$ & $-122,83$ \\
\hline Comunidad Valenciana & $-11,33$ & $-129,05$ & $-202,80$ & $-72,63$ \\
\hline Extremadura & $-6,72$ & $-9,62$ & $-30,90$ & $-29,18$ \\
\hline Galicia & $-23,30$ & $-53,55$ & $-68,28$ & $-8,75$ \\
\hline Madrid & $-16,90$ & $-72,35$ & $-180,33$ & $-41,92$ \\
\hline Murcia & 9,35 & $-28,50$ & $-73,05$ & $-5,45$ \\
\hline Navarra & $-3,73$ & $-6,10$ & $-19,25$ & $-2,22$ \\
\hline País Vasco & $-3,48$ & $-56,05$ & $-39,68$ & $-16,03$ \\
\hline Rioja (La) & $-1,23$ & $-9,15$ & $-6,38$ & $-7,43$ \\
\hline
\end{tabular}

Fuente: Elaboración propia.

El efecto total neto (Figura 3) muestra un comportamiento marcadamente diferente entre los dos periodos de estudio, sobre todo en el sector de la construcción. Se puede decir que en el periodo 2001-2006, se reduce, por lo general, el crecimiento en el empleo agrícola e industrial, a favor del sector de la construcción y los servicios, reflejándose esto en el crecimiento y competitividad de dichos sectores en la economía española. El sector servicios es el que muestra un comportamiento más favorable en el E.T.N., ya que ha presentado antes de la crisis valores positivos del mismo para catorce de las diecisiete CCAA, y en 2007-2012, para todas ellas. Este hecho se justifica gracias al crecimiento del 
empleo en este sector por encima de la media nacional (efecto sectorial positivo) para todas las CCAA en este periodo, incluso en comunidades en las que el sector servicios tiene menos peso relativo y no crece tan rápidamente como en las regiones vecinas (E.L.E. y E.C.N.E. negativos), como es el caso de Andalucía, Asturias o Extremadura -estos efectos se podrán observar detalladamente con posterioridad, en las Tablas 4 y 5 -.

Figura 3

Efecto total neto en los sectores económicos por CCAA (2001-2006 y 2007-2012)

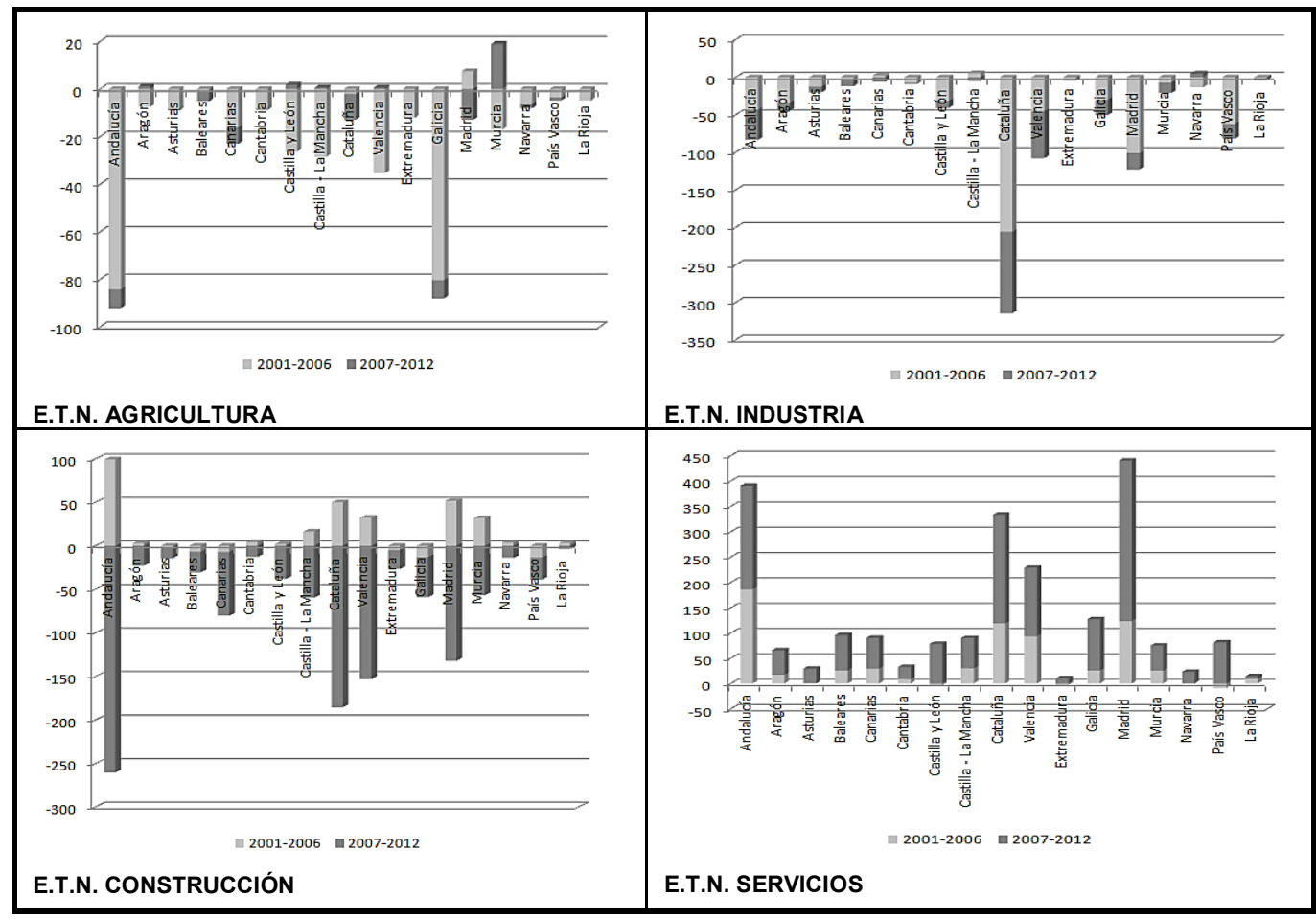

Fuente: Elaboración propia.

Durante la crisis aumenta el número de regiones en las que el empleo agrícola es más competitivo, aumentando también la especialización en el mismo en relación a los pesos relativos en las regiones vecinas (se ha pasado de sólo presentar un E.T.N. positivo en 2001-2006 la comunidad de Madrid, a cinco comunidades en esta situación en 2007-2012).

Sin embargo, en 2001-2006, en la construcción, más del 64\% de las CCAA presentaban un crecimiento en el empleo de este sector superior a la media nacional total (un total de once CCAA) y de éstas, nueve (Andalucía, Cantabria, 
Castilla-La Mancha, Cataluña, Valencia, Madrid, Murcia, Navarra y La Rioja) mostraban un crecimiento del empleo en el sector superior a la media de las regiones vecinas pero en tan sólo tres de ellas (Andalucía, Murcia y La Rioja), el efecto regional es superior al efecto sectorial. No obstante, en el siguiente periodo, en todas las CCAA el efecto total neto es negativo para el sector de la construcción, fundamentalmente motivado por un crecimiento más lento del empleo de este sector que el del conjunto de sectores de la nación.

A partir de los resultados recogidos en las Tablas 6 y 7 del Anexo (a partir de las cuales se han construido las Tablas 4 y 5 con el objeto de facilitar la interpretación de los diferentes efectos), se observa que la economía nacional "tira" o produce un efecto arrastre en el periodo 2001-2006 en todas las CCAA españolas en los cuatro sectores analizados, aunque este efecto se produce con mayor intensidad en el sector servicios (Duana, 2010:61). No obstante, en el periodo siguiente, el efecto nacional (E.N.) se vuelve negativo para todas las regiones y sectores, reflejo del decrecimiento de la economía nacional, cuya influencia se ve repercutida en todos los sectores económicos.

Tabla 4

Signos de los efectos del análisis shift-share espacialmente modificado para la población ocupada, 2001-2006

\begin{tabular}{|c|c|c|c|c|c|c|c|c|c|c|c|c|c|c|c|c|c|c|c|c|c|c|c|c|}
\hline & \multicolumn{6}{|c|}{ AGRICULTURA } & \multicolumn{6}{|c|}{ INDUSTRIA } & \multicolumn{6}{|c|}{ CONSTRUCCIÓN } & \multicolumn{6}{|c|}{ SERVICIOS } \\
\hline & \multirow{2}{*}{ EN } & \multirow{2}{*}{ Es } & \multicolumn{3}{|c|}{ ER } & \multirow{2}{*}{ ETN } & \multirow{2}{*}{ EN } & \multirow{2}{*}{ ES } & \multicolumn{3}{|c|}{ ER } & \multirow{2}{*}{ ETN } & \multirow{2}{*}{$\mathrm{N}$} & \multirow{2}{*}{ ES } & \multirow[b]{2}{*}{ TOT } & \multicolumn{2}{|l|}{$E R$} & \multirow{2}{*}{ ETN } & \multirow{2}{*}{\multicolumn{2}{|c|}{$\mathrm{EN}$}} & \multicolumn{3}{|c|}{ E.R. } & \\
\hline & & & TOTAL & ECNE & ELE & & & & TOTAL & ECNE & ELE & & & & & ECNE & ELE & & & & TOTAL & ECNE & & \\
\hline Andalucía & + & - & + & + & + & - & + & - & + & + & - & - & + & + & + & + & + & + & + & + & + & + & + & + \\
\hline Aragón & + & - & + & + & - & - & + & - & - & - & - & - & + & + & - & - & + & + & + & + & - & - & + & + \\
\hline Asturias & + & - & - & - & + & - & + & - & + & + & - & - & + & + & - & - & + & - & + & + & - & - & - & + \\
\hline Baleares & + & - & + & + & - & - & + & - & + & + & - & - & + & + & - & - & - & - & + & + & + & + & + & + \\
\hline Canarias & + & - & - & - & + & - & + & - & + & + & - & + & + & + & - & - & - & - & + & + & - & - & - & + \\
\hline Cantabria & + & - & - & - & + & - & + & - & - & - & + & - & + & + & + & + & + & + & + & + & + & + & + & + \\
\hline Castilla y León & + & - & + & + & + & - & + & - & + & + & - & - & + & + & - & - & - & + & + & + & - & - & + & - \\
\hline Castilla - La Mancha & + & - & - & - & - & - & + & - & + & + & - & + & + & + & + & + & + & + & + & + & + & + & - & + \\
\hline Cataluña & + & - & + & + & - & - & + & - & - & - & - & - & + & + & + & + & - & + & + & + & + & + & - & + \\
\hline Comunidad Valenciana & + & - & - & - & + & - & + & - & + & + & + & - & + & + & + & + & + & + & + & + & + & + & - & + \\
\hline Extremadura & + & - & + & + & + & - & + & - & + & + & - & - & + & + & - & - & - & - & + & + & - & - & - & - \\
\hline Galicia & + & - & - & - & - & - & + & - & + & + & - & - & + & + & - & - & - & - & + & + & - & - & + & + \\
\hline Madrid & + & - & + & + & - & + & + & - & - & - & + & - & + & + & + & + & - & + & + & + & + & + & \begin{tabular}{l|l}
+ \\
\end{tabular} & + \\
\hline Murcia & + & - & + & + & + & - & + & - & + & + & - & - & + & + & + & + & + & + & + & + & + & + & - & + \\
\hline Navarra & + & - & - & - & + & - & + & - & - & - & - & - & + & + & + & + & - & + & + & + & - & - & + & + \\
\hline País Vasco & + & - & + & + & - & - & + & - & - & - & - & - & + & + & - & - & + & - & + & + & - & - & - & - \\
\hline Rioja (La) & + & - & - & - & - & - & + & - & + & + & + & - & + & + & + & + & - & + & + & + & + & + & - & + \\
\hline
\end{tabular}

Fuente: Elaboración propia. 


\section{Tabla 5}

Signos de los efectos del análisis shift-share espacialmente modificado para la población ocupada, 2007-2012

\begin{tabular}{|c|c|c|c|c|c|c|c|c|c|c|c|c|c|c|c|c|c|c|c|c|c|c|c|c|}
\hline & \multicolumn{6}{|c|}{ AGRICULTURA } & \multicolumn{6}{|c|}{ INDUSTRIA } & \multicolumn{6}{|c|}{ CONSTRUCCIÓN } & \multicolumn{6}{|c|}{ SERVICIOS } \\
\hline & \multirow[b]{2}{*}{ EN } & & \multicolumn{3}{|c|}{ ER } & \multirow{2}{*}{ ETN } & \multirow{2}{*}{ EN } & \multirow[b]{2}{*}{ Es } & \multirow[b]{2}{*}{ TOT } & \multicolumn{2}{|l|}{ ER } & \multirow[b]{2}{*}{ ETN } & \multirow{2}{*}{ EN } & \multirow[b]{2}{*}{ Es } & \multirow[b]{2}{*}{ TOTA } & \multicolumn{2}{|l|}{$E R$} & \multirow[b]{2}{*}{ ETN } & \multirow{2}{*}{$\mathrm{EN}$} & \multirow[b]{2}{*}{ Es } & \multicolumn{3}{|c|}{ ER } & \\
\hline & & & TOTAL & ECNE & ELE & & & & & ECNE & ELE & & & & & ECNE & ELE & & & & TOTAL & ECNE & & \\
\hline Andalucía & - & - & + & + & + & - & - & - & - & - & + & - & - & -1 & - & - & - & - & - & + & - & - & - & + \\
\hline Aragón & - & - & + & + & - & + & - & - & + & + & + & - & - & - & + & + & - & - & - & + & + & + & - & + \\
\hline Asturias & - & - & + & + & - & - & - & - & + & + & + & - & - & - & + & + & - & - & - & + & - & - & - & + \\
\hline Baleares & - & - & - & - & + & - & - & - & - & - & + & - & - & -1 & + & + & + & - & - & + & + & + & + & + \\
\hline Canarias & - & - & - & - & + & - & - & - & - & - & + & - & - & -1 & - & - & - & - & - & + & - & - & - & + \\
\hline Cantabria & - & - & - & - & + & - & - & - & + & + & + & - & - & - & + & + & - & - & - & + & + & + & + & + \\
\hline Castilla y León & - & - & + & + & + & + & - & - & + & + & + & - & - & - & + & + & - & - & - & + & + & + & - & + \\
\hline Castilla - La Mancha & - & - & + & + & + & + & - & - & + & + & + & - & - & - & + & + & + & - & - & + & + & + & - & + \\
\hline Cataluña & - & - & - & - & + & - & - & - & - & - & - & - & - & - & + & + & - & - & - & + & - & - & - & + \\
\hline Comunidad Valenciana & - & - & + & + & - & + & - & - & - & - & - & - & - & - & - & - & - & - & - & + & - & - & + & + \\
\hline Extremadura & - & - & + & + & + & - & - & - & + & + & - & - & - & - & + & + & + & - & - & + & - & - & - & + \\
\hline Galicia & - & - & - & - & - & - & - & - & + & + & + & - & - & - & + & + & - & - & - & + & + & + & - & + \\
\hline Madrid & - & - & - & - & + & - & - & - & + & + & - & - & - & - & + & + & - & - & - & + & + & + & + & + \\
\hline Murcia & - & - & + & + & + & + & - & - & - & - & + & - & - & - & - & - & - & - & - & + & + & + & - & + \\
\hline Navarra & - & - & - & - & + & - & - & - & + & + & + & + & - & - & + & + & - & - & - & + & + & + & - & + \\
\hline País Vasco & \begin{tabular}{|l|}
- \\
\end{tabular} & - & - & - & + & - & - & - & + & + & + & - & - & - & + & + & - & - & - & + & + & + & + & + \\
\hline Rioja (La) & - & - & + & + & - & - & 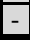 & - & + & + & + & - & - & - & + & + & - & - & - & + & - & - & + & + \\
\hline
\end{tabular}

Fuente: Elaboración propia.

Las CCAA en las que el Efecto Nacional alcanza valores superiores para cada sector, en periodo de crecimiento, coinciden, de forma general, con las que presentan los mayores valores negativos durante la crisis, por el efecto de arrastre económico comentado anteriormente. Éstas son, para el sector agrícola: Andalucía, Galicia y Castilla y León; para el sector industrial: Cataluña, Valencia y Madrid; en la construcción: Andalucía, Cataluña y Valencia y, finalmente, en el sector servicios: Madrid, Cataluña y Andalucía.

En lo que respecta al efecto sectorial o estructural (E.S.), los sectores agrícola e industrial se presentan como poco dinámicos respecto al resto de sectores tanto en épocas de crecimiento (2001-2006) como de crisis (2007-2012), ya que toman valores negativos para todas las CCAA en ambos periodos. Destacan por sus valores más elevados, regiones eminentemente agrícolas, en el primer caso, como Andalucía y Galicia, y más industriales, en el segundo caso, como Cataluña, Valencia o Madrid. Según la interpretación dada por Arias y Sánchez (2010:34), la negatividad del E.S., se puede atribuir a una alta concentración en industrias de poco crecimiento en el caso agrícola, por ejemplo, o a una débil concentración en industrias de rápido crecimiento.

Sin embargo, el sector de la construcción, que creció de forma exponencial en los años precedentes a la crisis, se presenta como un sector poco dinámico en todas las regiones en 2007-2012 (E.S. negativo), destacando Andalucía, Cataluña, Valencia y Madrid. Servicios es el único sector que ha presentado un efecto sectorial positivo para todo el espacio temporal y geográfico analizado, 
siendo por tanto un sector en el que el empleo crece más rápido que la media nacional.

En cuanto al efecto regional comparado (E.R.), sólo las comunidades de Andalucía y Murcia presentan un crecimiento del empleo en todos los sectores superior a la media de las regiones vecinas en el primer periodo. Sin embargo, durante la crisis, las regiones en las que se observa esta característica se modifican, pasando a ser Aragón, Castilla y León y Castilla-La Mancha.

A partir de la descomposición del E.R., teniendo en cuenta la influencia de las comunidades limítrofes en el empleo, se han obtenido el efecto competitivo neto espacial (E.C.N.E.) y el efecto locacional espacial (E.L.E.). Antes de la crisis, aquellas regiones más dinámicas y que estaban especializadas en ramas en las que eran más competitivas que las comunidades vecinas, eran: Andalucía, Castilla y León y Extremadura (en agricultura), Valencia y La Rioja (en industria), Andalucía, Cantabria, Castilla-La Mancha, Valencia y Murcia (en construcción) y Andalucía, Baleares y Madrid (en servicios). Sin embargo, durante la época de crisis, se observa una reducción del número de regiones más competitivas y especializadas que sus comunidades colindantes, tanto en el sector agrícola como en el industrial. Destaca, en el sector servicios, el incremento del número de regiones que durante la crisis han perdido dinámica competitiva, con un crecimiento del empleo en el sector menor que el nacional, pero un mayor peso relativo del empleo en ese sector en comparación con las regiones vecinas.

Es necesario comentar también la observación de la escasa influencia del E.L.E. para casi todas las CCAA y sectores en ambos periodos analizados, siendo, por tanto, más la competitividad (E.C.N.E.) que la especialización sectorial de cara región (E.L.E.) el componente de mayor influencia en la determinación del efecto regional. Este hecho también se ha observado en los trabajos de Duarte et al. (2000), Ramajo y Márquez (2008) y Arias y Sánchez (2010) pero sólo en algunos momentos del tiempo y sectores para los diferentes territorios estudiados y no de forma tan generalizada como se muestra en las Tablas 4 y 5 .

Con el objeto de profundizar en los efectos espaciales sobre el empleo en el sector en el que se centra esta investigación, la construcción, se han elaborado dos figuras para sendos periodos analizados, recogiendo en los mismos los componentes espaciales del análisis shift-share. El componente E.C.N.E. refleja la competitividad regional espacial, mientras que el componente E.L.E. se refiere a la especialización sectorial espacial (Figura 4 y Figura 5).

A partir de la Figura 4 se observa que, antes de la crisis, cinco CCAA tenían signo positivo en ambos componentes presentando un crecimiento del empleo en la construcción mayor que a nivel nacional, y una mayor participación de ese sector en sus economías en relación a sus regiones vecinas. De estas cinco regiones, durante la crisis, tan sólo una de ellas se mantuvo con ambos efectos 
positivos, Castilla-La Mancha, mientras que Valencia, Murcia y Andalucía han experimentado un efecto de inversión, presentando valores negativos en los componentes de competitividad y locacional. Por último, Cantabria ha perdido peso relativo del empleo en la construcción en relación a sus regiones limítrofes.

\section{Figura 4}

Descomposición del efecto regional para el sector de la construcción, 2001-2006

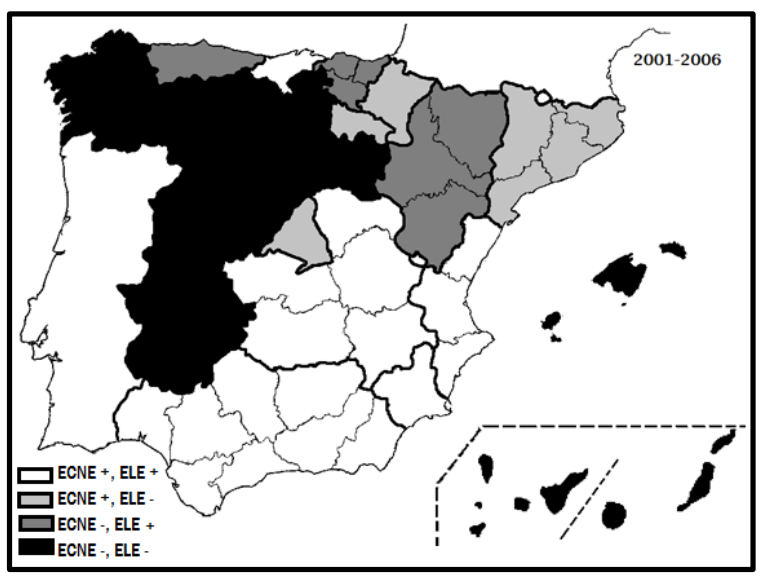

Fuente: Elaboración propia.

Figura 5

Descomposición del efecto regional para el sector de la construcción, 2007-2012

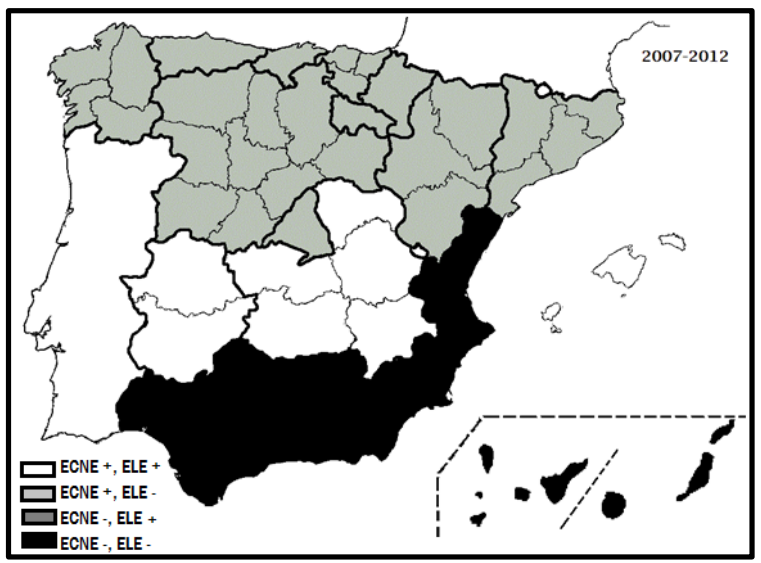

Fuente: Elaboración propia.

Asturias, País Vasco y Aragón se caracterizaban (en el periodo 2001-2006) por una menor competitividad en la construcción en relación a la nación, a lo 
que se unía una menor participación de dicho sector en sus economías, en relación a las comunidades limítrofes. Se observa que durante la crisis, ninguna Comunidad Autónoma se encuentra en esta situación (signo negativo en E.C.N.E. y positivo en E.L.E.) mientras que en las tres mencionadas, el empleo en la construcción crece a un ritmo mayor que a nivel nacional, a pesar de continuar con un menor peso relativo en el sector que las regiones vecinas (Figura $5)$.

En situación justamente inversa, se encuentran aquellas regiones son un signo negativo en E.C.N.E. y positivo en E.L.E.

Galicia, Castilla y León, Extremadura y las CCAA de Baleares y Canarias mostraban tanto el E.C.N.E. como el E.L.E. con ambos signos negativos reflejando una menor competitividad regional en la construcción respecto al sector nacional, y una mayor participación de ese sector en sus economías en relación a sus regiones vecinas. Situación en la que, a partir de la crisis, tan sólo se encuentra Canarias. Tanto Baleares como Extremadura han mejorado la competitividad de su empleo en construcción mientras que Galicia y Castilla y León han empeorado en ambos factores, competitivo y locacional.

Finalmente, las CCAA de Madrid, La Rioja, Navarra y Cataluña presentaban en ambos periodos un crecimiento del empleo en la construcción mayor que a nivel nacional, y un menor peso relativo del mismo en relación a sus regiones limítrofes.

Hay que mencionar en este sector un notable aumento de E.L.E. negativos en las diferentes CCAA para el periodo 2007-2012, explicados, fundamentalmente, por un mayor peso del empleo en la construcción en la región que en las que la rodean.

\section{CONCLUSIONES}

Seis años antes de la actual crisis en España, la economía de muchas de sus regiones se encontraban relativamente especializadas en sectores como el agrícola, construcción y servicios. Sin embargo, durante la crisis, el número de CCAA especializadas en estos dos últimos sectores ha descendido notablemente, al representar los mismos un mayor peso relativo en el empleo de la región pero crecer a un menor ritmo que en las comunidades limítrofes.

En términos generales, en el periodo 2001-2006 en España, el empleo en el sector agrícola e industrial decrece, a favor de los sectores de la construcción y servicios. Sin embargo, en el periodo 2007-2012, comunidades que presentaban antes de la crisis un mejor comportamiento en el empleo en la construcción y servicios que a nivel nacional, dejaron de ser competitivas en estos sectores, como es el caso de Andalucía o Valencia. 
El sector de la construcción se ha visto afectado por las fluctuaciones del ciclo económico de forma más intensa que el resto de sectores, creciendo por encima de la media nacional en la época de expansión y decreciendo más en la etapa de recesión (ha sido el único sector en España que ha experimentado tasas de crecimiento en sus ocupados negativas para todas las CCAA durante el periodo 2007-2012, además de los valores más elevados de las mismas, en relación al conjunto de la actividad económica).

En definitiva, la llegada de la crisis ha invertido muchas de las tendencias económicas precedentes y ha alterado el comportamiento de diversas variables, entre una de las más importantes se encuentra el empleo. Se puede decir que pocos años antes de 2007, eran los sectores de la construcción y servicios los que generaban un mayor crecimiento en el empleo. Sin embargo, tras la llegada de la crisis y su posterior desarrollo, se ha comprobado que en muchas regiones españolas, el empleo agrícola ha aumentado su crecimiento comparativamente al resto de sectores, mientras que éste es negativo para el sector de la construcción en toda España.

\section{REFERENCIAS BIBLIOGRÁFICAS}

ALAMÁ, L. y FUERTES, A.M. (2009). "Cronología de la crisis económica y debilidades de la globalización. La economía española en el contexto internacional”. Economía y Sociedad, 53.

ALONSO PÉREZ, M. y FURIO BLASCO, E. (2010). "La economía española: del crecimiento a la crisis pasando por la burbuja inmobiliaria". Cahiers de civilisation espagnole contemporaine, 6. Disponible en: http://ccec.revues.org/3212

ÁLVAREZ, J.A. (2008). "La banca española ante la crisis financiera". Estabilidad Financiera, 15, pp.21-38.

ÁlVAREZ ALEDO, C., DAVIA RODRÍGUEZ, M.A. y LEGAZPE MORALEJA, N. (2013). "Impacto laboral de la crisis económica: privación de empleo y precariedad". Papeles de economía española, 135, pp. 83-98.

ARCELUS, F. J. (1984). An Extension of shift-share Analysis. Growth and Change, 15, pp. 3-8.

ARIAS RAMÍREZ, R. y SÁNCHEZ HERNÁNDEZ, L. (2010). "Competitividad, especialización y mercado laboral en el cantón de Pérez Zeledón: Un análisis de economía regional”. IICE, 28(1).

ARIAS RAMÍREZ, R. y SÁNCHEZ HERNÁNDEZ, L. (2011). "Análisis de la dinámica regional del empleo utilizando el modelo shift-share espacialmente modificado: el caso de la región Chorotega, 1990, 2009". Ciencias Económicas, 29(2), pp. 399-418.

AYALA, L., CANTÓ, O. y MARTíNEZ, R. (2009). "El impacto de la crisis en la pobreza y la desigualdad”. En Laparra, M. y Pérez, B. (Coord.). El primer impacto de la crisis en la cohesión social en España. Colección Estudios, 32, pp. 21-57. 
BENTOLILA, S., CAHUC, P., DOLADO, J. y LE BARBANCHON, T. (2010). "Paro y empleo temporal durante la crisis: Una comparación entre Francia y España". En FEDEA. Disponible en: www.crisis09.es/libro_crisis/la_crisis_de_la_economia_ espanola.pdf [Último acceso: 3 de enero de 2014].

BERZEG, K. y KORAN, T. (1984). "A note on statistical approaches to shift-share analysis". Journal of Regional Science, 24(2), pp. 277-285.

BRENNER, R. (2002). "La economía mundial entra en recesión: un diagnóstico". Revista Herramienta, 19. http://www.herramienta.com.ar/revista-herramienta-n-19/ [Último acceso: 30 de diciembre de 2013].

CASE, A.C., ROSEN, H.S. y HINES, J.R. (1993). "Budget spillovers and fiscal policy interdependence: evidence from the states". Journal of Public Economics, 52, pp. 285-307.

CEBRIÁN LÓPEZ, I. y MORENO RAYMUNDO, G. (2012). "La estabilidad de los nuevos contratos indefinidos durante la crisis económica". Estudios de Economía Aplicada, 30(1), pp. 183-208.

CLIFF, A. y ORD, J.K. (1973). Spatial autocorrelation. London: Pion.

CASLER, S.D. (1989). "A theorical context for shift and share analysis". Regional Studies, 23(1), pp. 43-48.

CHALMERS, J.A. y BECKHELM, T.L. (1976). "Shift and share and the theory of industrial location". Regional Studies, 4(1), pp. 15-23.

CONDE RUIZ, J.I., FELGUEROSO, F. y GARCÍA PÉREZ, J.I. (2011). "Reforma laboral 2010: una primera evaluación y propuestas de mejora". Revista de Economía Aplicada, 57, pp. 147-180.

CONSEJO ECONÓMICO Y SOCIAL DE ESPAÑA (2013). Informe sobre distribución de la renta en España: desigualdad, cambios estructurales y ciclos, aprobado en la Sesión ordinaria del Pleno de 20 de febrero de 2013. Comisión de Trabajo de Economía y Fiscalidad.

CÖRVERS, F. y MERIKÜLL, J. (2007). "Occupational structures across 25 EU countries: the importance of industry structure and technology in old and new EU countries". Economic Change, 40, pp. 327 - 359.

CUERVO CAZURRA, A. (2008). "Las distancias en el proceso de internacionalización: el caso del Banco Santander". Universia Business Review, núm. Extra 17, pp. 96-111.

DAWSON, J. (1982). "shift-share Analysis: a Bibliographic Review of Technique and Applications". Vance Bibliographies, Monticello, Illinois.

DE LA DEHESA ROMERO, G. (2003). "Balance de la economía española en los últimos veinticinco años", ICE: Revista de Economía, 811, pp. 53-79.

DELGADO RIVERO, F.J., LÓPEZ MENÉNDEZ, A.J. y PÉREZ SUÁREZ, R. (1997). "Dinámica de las economías regionales 1986-1996. El caso de Asturias", Documento de trabajo Hispalink-Asturias, 2, pp. 1-28.

DINC, M.; HAYNES, K. y QIANGSHENG, L. (1998). "A comparative evaluation of shiftshare models and their extensions". Australasian Journal of Regional Studies, 52 (1), pp. 275-302.

DUANA ÁVILA, D. (2010). "Excedentes petroleros en el crecimiento de México: una aplicación de la técnica shift-share 2003-2004". Suma de Negocios, 1(2), pp. 57-72.

DUARTE, R., ESCARIO, J. J. y MUR, M. (2000). "Descomposición del crecimiento económico: Una aplicación de la técnica Shift-Share a la Comunidad Aragonesa", VII Jornadas de Economía Crítica, Albacete. 
DUNN, E. (1960). "A statistical and analytical technique for regional analysis". Papers of the Regional Science Association, 6, pp. 97-112.

ESTEBAN-MARQUILLAS, J. M. (1972). "A reinterpretation of shift-share analysis". Regional and Urban Economics, 2(3), pp. 249-255.

ESTEBAN TALAYA, A.; MONDÉJAR JIMÉNEZ, J.A.; MONDÉJAR JIMÉNEZ, J., y MESEGUER EZCURRA, R. y RAPÚN, M. (2006). "Regional Disparities and National Development Revisited the Case of Western Europe". European Urban and Regional Studies, 13, pp. 355-369.

EUROSTAT. http://epp.eurostat.ec.europa.eu/portal/page/portal/statistics/themes [Último acceso: 10 de enero de 2014].

FERNÁNDEZ, A. y CRUZ, E. (2013). "Análisis territorial del crecimiento y la crisis del sector de la construcción en España y la Comunidad Autónoma de Andalucía”. Eure, 39(116), pp. 5-37.

FERNÁNDEZ DE LIS, S. y GARCÍA MORA, A. (2008). "Algunas implicaciones de la crisis financiera sobre la banca minorista española". Estabilidad financiera, 15, pp. 55-72.

FOTHERGILL, S. y GUDGIN, G. (1979). "In defence of shift-share". Urban Studies, 16(3), pp. 309-319.

FOTOPOULOS, G. (2007). "Integrating Firm Dynamics into the shift-share Framework", Growth and Change, 38, pp. 140-152.

FUCHS, V.R. (1959). "Changes in the location of U.S. manufacturing since 1929". Journal of Regional Science, 1(2), pp. 1-17.

FUNDACIÓN ALTERNATIVAS (2013). Primer informe sobre la desigualdad en España. Bubok.

GARCÍA MONTALVO, J. (2009). "Financiación inmobiliaria, burbuja crediticia y crisis financiera, Lecciones a partir de la recesión de 2008-09.". Papeles de Economía Española, 122, pp. 66-87.

GARCÍA SERRANO, C. (2011). "Déjà vu? Crisis de empleo y reformas laborales en España". Revista de Economía Aplicada, 56, pp. 149-177.

GARCÍA SERRANO, C. (2012). "Del pasmo al marasmo. El sector de la construcción y su relación con la crisis del empleo". Estudios de Economía Aplicada, 30(1), pp. 163182.

GARRIDO, R. Y. (2002). Cambio estructural y desarrollo regional en España, Madrid: Editorial Pirámide.

GERKING, S.D. y BARRINGTON, J. (1981). "Are regional share effects constant over time?". Journal of Regional Science, 21, pp. 163-174.

GHEMAWAT, P. (2001). "Distance Still Matters". Harvard Business Review, 79(8), pp. 137-145.

GRADIN, C., CANTÓ, O. y DEL RÍO, C. (2012). "Measuring employment deprivation among households in the EU". ECINEQ Working Papers Series, 247, Society for the Study of Economic Inequality. Disponible en: http://www.ecineq.org/milano/WP/ ECINEQ2012-247.pdf [Último acceso: 2 de enero de 2014).

GRAHAM, D. y SPENCE, N. (1998). "A productivity growth interpretation of the labour demand shift-share model”. Regional Studies, 32(6), pp. 515-525. 
HEWINGS, G.J.D. (1976). "On the accuracy of alternative models for stepping-down multicounty employment projections to counties". Economic Geography, 52, pp. 206217.

INSTITUTO NACIONAL DE ESTADÍSTICA (INE). http://www.ine.es/ [Último acceso: 6 de octubre de 2013].

INSTITUTO VALENCIANO DE INVESTIGACIONES ECONÓMICAS (IVIE) (2011). Capital humano y empleo en tiempos de crisis, $n^{\circ}$ 122. http://www.ivie.es/downloads/ docs/ch/ch122.pdf [Último acceso: 9 de enero de 2014].

ISARD, W. (1960). Methods of Regional Analysis: an Introduction to Regional Science, New York: The Technology Press of Massachusetts Institute of Technology and John Wiley and Sons, Inc.

IZQUIERDO, M. y REGIL, A.V. (2006). "Actualización de las estimaciones de la tasa de desempleo estructural de la economía española". Boletín Económico, 9, pp. 55-62.

KLUGMAN, J., RODRÍGUEZ, F. y CHOI, H-J. (2011). "The HDI 2010: new controversies, old critiques". Journal of Economic Inequality, 9, pp. 249-288.

KNUDSEN, D.C. y BARF, R. (1991). "Shift-Share Analysis as a Linear Model". Environment and Planning, 23, pp. 421-431.

LA CAIXA (2012). "Informe mensual”. Estudios y análisis económico, 354.

LÓPEZ ZAFRA, J.M. (2013). "Empleo y crisis económica. Una visión global”. En CoscuIluela Martínez, C., de Paz Cobo, S. y Riobóo Lestón, I. (Coord.). La situación actual del mercado laboral (pp. 41-49). Madrid: Editorial del Economista.

LOVERIDGE, S. y SELTING, A.C. (1998). "A Review and Comparison of shift-share Identities". International Regional Science Review, 21, pp. 37-58.

MAYOR FERNÁNDEZ, M. y LÓPEZ MENÉNDEZ, A.J. (2005). "El análisis shift-share: nuevos desarrollos espaciales". VIII Encuentro de Economía Aplicada, Murcia.

MEDINA, E.; HERRARTE, A. y VICÉNS, J. (2010). "Inmigración y desempleo en España: impacto de la crisis económica”. ICE, 854, pp. 37-48.

MINISTERIO DE EDUCACIÓN, CULTURA Y DEPORTE. Las cifras de la educación en España. Varios cursos. http://www.mecd.gob.es/portada-mecd/ [Último acceso: 6 de octubre de 2013].

MORAL CARCEDO, J., GARCÍA-BELENGUER CAMPOS, F. y BOTE ÁLVAREZ-CARRASCO, V. (2012). "Flexibilidad del tiempo de trabajo en España: ¿Ha alterado la crisis el comportamiento del empleo a tiempo parcial?". Estudios de Economía Aplicada, 30(1), pp. 209-236.

MORENO SERRANO, R. y VAYÁ VALCARCE, E. (2000). Técnicas econométricas para el tratamiento de datos espaciales: la econometría espacial. Edicions Universitat Barcelona.

MUÑOZ DE BUSTILLO, R.L. y ANTÓN, J.I. (2011). "From the highest employment growth to the deepest fall: Economic crisis and labour inequalities in Spain". En Vaughanwhitehead, D. (Ed.). Work inequalities in the crisis. Evidence from Europe (pp.393-444). OIT Cheltenham: Edward Elgar.

MUR LACAMBRA, J. (1992). "Contrastes de autocorrelación espacial. Un estudio de Monte Carlo”. Estadística española, 34(130), pp. 285-307.

NAVARRO ARANCEGUI, M. (2008). El entorno económico y la competitividad en España. Bilbao: Universidad de Deusto. 
NAVARRO, V., TORRES, J. y GARZÓN, A. (2011). Hay alternativas. Propuestas para crear empleo y bienestar social en España. Madrid: Sequitur.

NAZARA, S. y HEWINGS, G.J.D. (2004). "Spatial Structure and Taxonomy of Decomposition in shift-share analysis". Growth and Change, 35(4), pp. 476 - 490.

ORDÓÑEZ GALÁN, C., VARELA GONZÁLEZ, M.R. y REYES PANTOJA, A. (2011). "Desarrollo de un SIG para el análisis de patrones espaciales de incendios en viviendas", GeoFocus, pp. 1-18.

ORGANIZACIÓN DE NACIONES UNIDAS (ONU) (2013). Informe sobre Desarrollo Humano 2013. Programa de las Naciones Unidas.

ORTEGA, E. y PEÑALOSA, J. (2012). Claves de la crisis económica española y retos para crecer en la UEM. Documentos ocasionales, 1201, Banco de España.

PAJARES, M. (2007). Inmigración y mercado de trabajo. Informe 2007. Análisis de datos de España y Cataluña. Madrid: Documentos del Observatorio Permanente de la Inmigración.

PEÑA, A.R. (2011). "Desarrollo económico regional en España: Análisis de la productividad y del empleo como factores determinantes". Boletín Económico del ICE (Información Comercial Española), 3007, pp. 39-48.

PEÑA, A.R. y JIMÉNEZ, M. (2013). "Productividad y estructura sectorial: elementos determinantes de las disparidades económicas regionales en España". Revista de Estudios Regionales, 97, pp.137-169.

PÉREZ INFANTE, J.I. (2006). Las estadísticas del mercado de trabajo en España. Madrid: Colección Informes y Estudios, 28.

RAMAJO HERNÁNDEZ, J. y MÁRQUEZ PANIAGUA, M.A. (2008). "Componentes espaciales en el modelo shift-share. Una aplicación al caso de las regiones peninsulares españolas", Estadística española, 50(168), pp. 247-272.

RECIO RAPÚN, M.L. (2013). "El mercado de trabajo español: una visión de conjunto". En Cosculluela Martínez, C., de Paz Cobo, S. y Riobóo Lestón, I. (Coord.). La situación actual del mercado laboral (pp. 51-72). Madrid: Editorial del Economista.

RICHARDSON, H. (1978). "The state of regional economics: A survey article". International Regional Science Review, 16(1), pp. 1-48.

RODRÍGUEZ LÓPEZ, J. (2007). “Urbanismo, vivienda y economía en España”. Revista ACE, 1(3), pp. 83-88.

SAKASHITA, N. (1973). "An econometric model of internal migration and development". Regional Science and Urban Economics, 14, pp. 77-87.

SÁNCHEZ DOMÍNGUEZ, M.A. y GARCÍA QUERO, F. (2011). "Las crisis económicas en España bajo una retrospectiva institucional: la historia importa". X Congreso internacional de la AEHE, 8-10 de septiembre de 2011. Universidad Pablo de Olavide, Sevilla.

SANROMÀ MELÉNDEZ, E. (2012). "El mercado de trabajo español en la crisis económica (2008-2012): desempleo y reforma laboral". Revista de Estudios Empresariales. Segunda época, 2, pp. 29-57.

THEIL, H. y GOSH, R. (1980). "A comparison of Shift Share analysis and the RAS Adjustment". Regional Science and Urban Economics, 10, pp. 175-180.

TOBLER, W. (1979). "Cellular geography”, en Gale, S. y G. Olsson (eds.): Philosophy in Geography (pp.379-386). Dordrecht: Reidel. 
VV.AA. (2013). Desigualdad y ruptura de la cohesión social. Economistas sin fronteras. Dossieres EsF, 9.

WADLEY, D. y SMITH, P. (2003). "Straightening up shift-share analysis", The Annals of Regional Science, 37, pp. 259-261.

YÁNEZ CONTRERAS, M. y ACEVEDO GONZÁLEZ, K. (2010). "El mercado laboral desde una perspectiva espacial”. Apuntes del CENES, 50, pp. 73-97. 


\section{ANEXO}

Tabla 6

Efectos del análisis shift-share espacialmente modificado para la población ocupada, 2001-2006

\begin{tabular}{|c|c|c|c|c|c|c|c|c|c|c|c|c|}
\hline & \multicolumn{6}{|c|}{ AGRICULTURA } & \multicolumn{6}{|c|}{ INDUSTRIA } \\
\hline & \multirow{2}{*}{ E.N. } & \multirow{2}{*}{ E.S. } & \multicolumn{3}{|c|}{ E.R. } & \multirow{2}{*}{ E.T.N. } & \multirow{2}{*}{ E.N. } & \multirow{2}{*}{ E.S. } & \multicolumn{3}{|c|}{ E.R. } & \multirow{2}{*}{ E.T.N. } \\
\hline & & & TOTAL & E.C.N.E. & E.L.E. & & & & TOTAL & E.C.N.E. & E.L.E. & \\
\hline Andalucía & 61,94 & $-88,61$ & 4,34 & 3,08 & 1,27 & $-84,26$ & 65,61 & $-54,86$ & 11,88 & 20,14 & $-8,26$ & $-42,98$ \\
\hline Aragón & 8,21 & $-11,74$ & 4,21 & 4,56 & $-0,35$ & $-7,53$ & 28,61 & $-23,92$ & $-9,84$ & $-7,75$ & $-2,09$ & $-33,76$ \\
\hline Asturias & \begin{tabular}{|l|}
5,84 \\
\end{tabular} & $-8,35$ & $-0,06$ & $-0,07$ & 0,01 & $-8,41$ & 16,55 & \begin{tabular}{|l|}
$-13,84$ \\
\end{tabular} & 1,21 & 1,23 & $-0,02$ & $\mid-12,63$ \\
\hline Baleares & 1,74 & $-2,50$ & 1,80 & 7,40 & $-5,60$ & $-0,69$ & 8,48 & $-7,09$ & 2,59 & 5,54 & $-2,95$ & $-4,50$ \\
\hline Canarias & 8,34 & $-11,94$ & $-4,13$ & $-6,42$ & 2,29 & $-16,07$ & 10,85 & $-9,07$ & 11,27 & 34,13 & $-22,86$ & 2,20 \\
\hline Cantabria & 3,42 & $-4,90$ & $-3,40$ & $-3,75$ & 0,34 & $-8,30$ & 9,37 & $-7,84$ & $-0,04$ & $-0,04$ & 0,00 & $-7,87$ \\
\hline Castilla y León & 20,31 & $-29,06$ & 2,65 & 2,14 & 0,51 & $-26,41$ & 39,73 & $-33,22$ & 1,82 & 1,91 & $-0,09$ & $-31,40$ \\
\hline Castilla - La Mancha & 15,86 & $-22,69$ & $-5,77$ & $-4,19$ & $-1,58$ & $-28,46$ & 27,09 & $-22,65$ & 28,01 & 30,18 & $-2,17$ & 5,36 \\
\hline Cataluña & 16,33 & $-23,36$ & 21,31 & 67,06 & $-45,75$ & $-2,05$ & 181,77 & $-152,00$ & $-53,75$ & $-38,52$ & $-15,23$ & $-205,74$ \\
\hline Comunidad Valenciana & 18,05 & $-25,82$ & $-9,55$ & $-16,58$ & 7,02 & $-35,38$ & 90,75 & $-75,89$ & 30,64 & 26,80 & 3,83 & $-45,25$ \\
\hline Extremadura & 11,07 & $-15,84$ & 3,77 & 2,12 & 1,64 & $-12,07$ & 8,50 & $-7,11$ & 4,73 & 8,81 & $-4,08$ & $-2,37$ \\
\hline Galicia & 34,96 & $-50,02$ & $-30,37$ & $-16,29$ & $-14,08$ & $-80,39$ & 44,58 & $-37,28$ & 7,35 & 7,83 & $-0,49$ & $-29,93$ \\
\hline Madrid & 4,12 & \begin{tabular}{|l|}
$-5,89$ \\
\end{tabular} & 13,32 & 139,32 & $-126,00$ & 7,43 & \begin{tabular}{|l|}
81,51 \\
\end{tabular} & $-68,16$ & $-32,90$ & $-44,03$ & 11,13 & $-101,06$ \\
\hline Murcia & 13,89 & $-19,87$ & 2,78 & 1,68 & 1,10 & $-17,09$ & 18,23 & \begin{tabular}{|l|}
$-15,24$ \\
\end{tabular} & 7,79 & 9,09 & $-1,30$ & $-7,46$ \\
\hline Navarra & 3,62 & $-5,18$ & $-1,19$ & $-1,46$ & 0,27 & $-6,37$ & \begin{tabular}{|l|}
15,64 \\
\end{tabular} & \begin{tabular}{|l|}
$-13,07$ \\
\end{tabular} & $-0,29$ & $-0,21$ & $-0,08$ & \begin{tabular}{|l|}
$-13,36$ \\
\end{tabular} \\
\hline País Vasco & 3,40 & $-4,86$ & 1,29 & 6,05 & $-4,76$ & $-3,57$ & 55,52 & $-46,43$ & \begin{tabular}{|l|}
$-15,72$ \\
\end{tabular} & $-11,44$ & $-4,28$ & $-62,14$ \\
\hline Rioja (La) & 2,64 & $-3,78$ & $-0,99$ & $-0,76$ & $-0,22$ & $-4,76$ & 7,31 & $-6,12$ & 5,23 & 3,69 & 1,53 & $-0,89$ \\
\hline
\end{tabular}

\begin{tabular}{|c|c|c|c|c|c|c|c|c|c|c|c|c|}
\hline & \multicolumn{6}{|c|}{ CONSTRUCCIÓN } & \multicolumn{6}{|c|}{ SERVICIOS } \\
\hline & \multirow{2}{*}{ E.N. } & \multirow{2}{*}{ E.S. } & \multicolumn{3}{|c|}{ E.R. } & \multirow{2}{*}{ E.T.N. } & \multirow{2}{*}{ E.N. } & \multirow{2}{*}{ E.S. } & \multicolumn{3}{|c|}{ E.R. } & \multirow{2}{*}{ E.T.N. } \\
\hline & & & TOTAL & E.C.N.E. & E.L.E. & & & & TOTAL & E.C.N.E. & E.L.E. & \\
\hline Andalucía & 68,49 & 40,57 & 58,51 & 54,45 & 4,07 & 99,08 & 343,67 & 104,40 & 81,61 & 76,19 & 5,41 & 186,01 \\
\hline Aragón & 10,36 & 6,13 & $-3,37$ & $-4,20$ & 0,83 & 2,77 & 62,16 & 18,88 & $-1,20$ & $-1,25$ & 0,05 & 17,69 \\
\hline Asturias & 9,24 & 5,47 & $-7,24$ & $-7,54$ & 0,31 & $-1,76$ & 49,90 & 15,16 & $-14,79$ & $-14,36$ & $-0,42$ & 0,37 \\
\hline Baleares & 14,43 & 8,55 & $-15,52$ & $-11,20$ & $-4,33$ & $-6,98$ & 63,48 & 19,28 & 6,73 & 5,56 & 1,18 & 26,02 \\
\hline Canarias & 23,98 & 14,20 & $-21,26$ & $-16,69$ & $-4,57$ & $-7,05$ & 116,23 & 35,31 & $-5,98$ & $-4,88$ & $-1,10$ & 29,32 \\
\hline Cantabria & 5,73 & 3,39 & 0,53 & 0,51 & 0,02 & 3,92 & 27,83 & 8,45 & 0,00 & 0,00 & 0,00 & 8,45 \\
\hline Castilla y León & 24,63 & 14,59 & $-12,84$ & $-12,44$ & $-0,40$ & 1,75 & 117,32 & 35,64 & $-38,94$ & $-39,86$ & 0,92 & $-3,30$ \\
\hline Castilla - La Mancha & 20,85 & 12,35 & 3,93 & 3,15 & 0,78 & 16,28 & 77,82 & 23,64 & 6,57 & 7,10 & $-0,54$ & 30,21 \\
\hline Cataluña & 65,94 & 39,06 & 10,75 & 12,17 & $-1,42$ & 49,81 & 368,05 & 111,81 & 7,02 & 7,17 & $-0,15$ & 118,83 \\
\hline Comunidad Valenciana & 48,83 & 28,92 & 3,12 & 2,91 & 0,21 & 32,04 & 227,62 & 69,15 & 24,21 & 24,36 & $-0,15$ & 93,35 \\
\hline Extremadura & 11,50 & 6,81 & $-11,61$ & $-9,15$ & $-2,46$ & $-4,80$ & 45,70 & 13,88 & $-16,26$ & $-16,24$ & $-0,02$ & $-2,37$ \\
\hline Galicia & 27,78 & 16,46 & $-29,49$ & $-28,90$ & $-0,58$ & $-13,03$ & 123,29 & 37,45 & $-11,45$ & $-12,73$ & 1,28 & 26,01 \\
\hline Madrid & 49,72 & 29,45 & 21,85 & 27,46 & $-5,62$ & 51,30 & 394,02 & 119,70 & 3,71 & 2,96 & 0,75 & 123,41 \\
\hline Murcia & 12,48 & 7,39 & 24,25 & 23,69 & 0,56 & 31,64 & 58,65 & 17,82 & 7,83 & 8,20 & $-0,37$ & 25,65 \\
\hline Navarra & 4,99 & 2,95 & 0,56 & 0,72 & $-0,17$ & 3,51 & 30,56 & 9,28 & $-9,24$ & $-9,85$ & 0,61 & 0,04 \\
\hline País Vasco & 17,67 & 10,47 & $-23,81$ & $-31,21$ & 7,40 & $-13,35$ & 119,54 & 36,31 & $-45,25$ & $-44,14$ & $-1,11$ & $-8,94$ \\
\hline Rioja (La) & 2,26 & 1,34 & 1,63 & 2,13 & $-0,51$ & 2,97 & 12,86 & 3,91 & 5,43 & 6,29 & $-0,86$ & 9,34 \\
\hline
\end{tabular}

Fuente: Elaboración propia. 
Tabla 7

Efectos del análisis shift-share espacialmente modificado para la población ocupada, 2007-2012

\begin{tabular}{|c|c|c|c|c|c|c|c|c|c|c|c|c|}
\hline & \multicolumn{6}{|c|}{ AGRICULTURA } & \multicolumn{6}{|c|}{ INDUSTRIA } \\
\hline & \multirow{2}{*}{ E.N. } & \multirow{2}{*}{ E.S. } & \multicolumn{3}{|c|}{ E.R. } & \multirow{2}{*}{ E.T.N. } & \multirow{2}{*}{ E.N. } & \multirow{2}{*}{ E.S. } & \multicolumn{3}{|c|}{ E.R. } & \multirow{2}{*}{ E.T.N. } \\
\hline & & & TOTAL & E.C.N.E & E.L.E. & & & & TOTAL & E.C.N.E. & E.L.E. & \\
\hline Andalucía & $-37,70$ & $-8,71$ & 0,86 & 0,70 & 0,16 & $-7,85$ & $-49,23$ & $-33,69$ & $-6,96$ & $-11,41$ & 4,46 & $-40,64$ \\
\hline Aragón & $-5,39$ & $\mid-1,24$ & 2,31 & 2,51 & $-0,20$ & 1,06 & $-19,20$ & $-13,14$ & 2,19 & 1,75 & 0,44 & $-10,95$ \\
\hline Asturias & $-3,06$ & $\mid-0,71$ & 0,04 & 0,06 & $-0,01$ & $-0,67$ & $-11,25$ & $-7,70$ & 0,15 & 0,15 & 0,01 & $-7,55$ \\
\hline Baleares & $-1,67$ & $-0,39$ & $-4,07$ & $-11,89$ & 7,82 & $-4,46$ & $-7,38$ & $-5,05$ & $-2,65$ & $-4,58$ & 1,93 & $-7,70$ \\
\hline Canarias & $-5,03$ & $-1,16$ & $-5,84$ & $-10,20$ & 4,36 & $-7,00$ & $-8,21$ & $-5,62$ & $-0,77$ & $-2,14$ & 1,38 & $-6,39$ \\
\hline Cantabria & \begin{tabular}{|l|}
$-1,44$ \\
\end{tabular} & $-0,33$ & $-0,15$ & $-0,26$ & 0,11 & $-0,48$ & $-6,93$ & $-4,74$ & 3,35 & 3,13 & 0,22 & $-1,39$ \\
\hline Castilla y León & $-12,06$ & $-2,78$ & 4,52 & 3,86 & 0,66 & 1,73 & $-28,66$ & $-19,61$ & 9,92 & 9,31 & 0,60 & $-9,69$ \\
\hline Castilla - La Mancha & \begin{tabular}{|l|}
$-9,06$ \\
\end{tabular} & $-2,09$ & 2,63 & 2,35 & 0,28 & 0,54 & $-21,89$ & \begin{tabular}{|l|}
$-14,98$ \\
\end{tabular} & 9,86 & 9,53 & 0,33 & $-5,11$ \\
\hline Cataluña & $-11,59$ & $-2,68$ & $-8,18$ & $-23,74$ & 15,56 & $-10,86$ & $-115,32$ & $-78,91$ & $-29,87$ & $-22,81$ & $-7,06$ & $-108,78$ \\
\hline Comunidad Valenciana & $-11,83$ & $-2,73$ & 3,24 & 5,83 & $-2,59$ & 0,51 & $-66,40$ & $-45,43$ & $-17,22$ & $-14,45$ & $-2,77$ & $-62,65$ \\
\hline Extremadura & \begin{tabular}{|l|}
$-6,70$ \\
\end{tabular} & $-1,55$ & 1,53 & 0,90 & 0,63 & $-0,02$ & $-7,29$ & \begin{tabular}{|l|}
$-4,99$ \\
\end{tabular} & 2,66 & 3,78 & $-1,11$ & $-2,33$ \\
\hline Galicia & $-15,60$ & $-3,60$ & $-4,10$ & $-3,01$ & $-1,09$ & $-7,70$ & $-32,88$ & $-22,50$ & 1,82 & 1,66 & 0,16 & $-20,67$ \\
\hline Madrid & $-3,92$ & $-0,90$ & $-12,08$ & $-90,22$ & 78,14 & $-12,98$ & $-50,70$ & $-34,69$ & 13,03 & 19,69 & $-6,66$ & $-21,65$ \\
\hline Murcia & \begin{tabular}{|l|}
$-9,54$ \\
\end{tabular} & $-2,20$ & 21,09 & 13,42 & 7,67 & 18,89 & $-14,88$ & $-10,18$ & $-3,43$ & $-3,66$ & 0,23 & $-13,62$ \\
\hline Navarra & \begin{tabular}{|l|}
$-1,96$ \\
\end{tabular} & $-0,45$ & $-1,32$ & $-1,87$ & 0,56 & $-1,77$ & $-10,89$ & \begin{tabular}{|l|}
$-7,45$ \\
\end{tabular} & 12,24 & 8,17 & 4,07 & 4,79 \\
\hline País Vasco & $-2,25$ & $-0,52$ & $-0,71$ & $-2,98$ & 2,27 & $-1,22$ & $-36,23$ & $-24,79$ & 4,98 & 3,41 & 1,56 & $-19,82$ \\
\hline Rioja (La) & \begin{tabular}{|l|}
$-1,18$ \\
\end{tabular} & $-0,27$ & 0,23 & 0,27 & $-0,04$ & $-0,04$ & $-5,83$ & $-3,99$ & 0,67 & 0,42 & 0,25 & $-3,32$ \\
\hline
\end{tabular}

\begin{tabular}{|c|c|c|c|c|c|c|c|c|c|c|c|c|}
\hline & \multicolumn{6}{|c|}{ CONSTRUCCIÓN } & \multicolumn{6}{|c|}{ SERVICIOS } \\
\hline & \multirow{2}{*}{ E.N. } & \multirow{2}{*}{ E.S. } & \multicolumn{3}{|c|}{ E.R. } & \multirow{2}{*}{ E.T.N. } & \multirow{2}{*}{ E.N. } & \multirow{2}{*}{ E.S. } & \multicolumn{3}{|c|}{ E.R. } & \multirow{2}{*}{ E.T.N } \\
\hline & & & TOTAL & E.C.N.E. & E.L.E. & & & & TOTAL & E.C.N.E & E.L.E. & \\
\hline Andalucía & $-74,26$ & $-208,13$ & $-52,36$ & $-48,58$ & \begin{tabular}{|l|}
$-3,78$ \\
\end{tabular} & $-260,49$ & $-325,76$ & 242,47 & $-37,93$ & $-35,68$ & $-2,25$ & 204,54 \\
\hline Aragón & $-10,69$ & $-29,96$ & 6,98 & 8,53 & $-1,55$ & $-22,98$ & $-57,10$ & 42,50 & 5,70 & 5,80 & $-0,10$ & 48,20 \\
\hline Asturias & $-7,71$ & $-21,61$ & 8,95 & 10,76 & \begin{tabular}{|l|}
$-1,81$ \\
\end{tabular} & \begin{tabular}{|l|}
$-12,66$ \\
\end{tabular} & \begin{tabular}{|l|}
$-43,53$ \\
\end{tabular} & 32,40 & $-3,12$ & $-2,96$ & $-0,16$ & 29,28 \\
\hline Baleares & $-12,19$ & $-34,17$ & 10,46 & 9,33 & \begin{tabular}{|l|}
1,13 \\
\end{tabular} & $\mid-23,71$ & $-55,60$ & 41,38 & 28,04 & 24,39 & 3,66 & 69,43 \\
\hline Canarias & $-21,19$ & $-59,39$ & $\mid-14,02$ & $-12,96$ & \begin{tabular}{|l|}
$-1,06$ \\
\end{tabular} & \begin{tabular}{|l|}
$-73,41$ \\
\end{tabular} & $\mid-104,03$ & 77,43 & $-16,68$ & $-13,97$ & $-2,71$ & 60,76 \\
\hline Cantabria & $-5,30$ & \begin{tabular}{|l|}
$-14,86$ \\
\end{tabular} & 2,11 & 2,20 & $-0,09$ & $-12,75$ & $-25,42$ & 18,92 & 5,43 & 5,25 & 0,18 & 24,35 \\
\hline Castilla y León & $-20,62$ & $-57,80$ & 19,88 & 22,13 & $-2,25$ & $-37,93$ & $-100,97$ & 75,15 & 3,19 & 3,23 & $-0,04$ & 78,35 \\
\hline Castilla - La Mancha & $-21,53$ & $-60,35$ & 0,73 & 0,61 & 0,12 & $-59,62$ & $-75,05$ & 55,86 & 3,74 & 4,00 & $-0,26$ & 59,60 \\
\hline Cataluña & $-66,48$ & $-186,32$ & 0,70 & 0,79 & \begin{tabular}{|l|}
$-0,09$ \\
\end{tabular} & $-185,62$ & $-337,62$ & 251,30 & $-36,50$ & $-36,13$ & $-0,37$ & 214,80 \\
\hline Comunidad Valenciana & $-49,60$ & $-139,01$ & $-14,19$ & $-13,60$ & $-0,59$ & $-153,20$ & $-208,05$ & 154,86 & $-19,43$ & $-19,74$ & 0,31 & 135,43 \\
\hline Extremadura & $-9,06$ & \begin{tabular}{|l|}
$-25,39$ \\
\end{tabular} & 3,55 & 3,46 & 0,09 & $-21,84$ & $-39,34$ & 29,28 & $-19,12$ & $-19,08$ & $-0,04$ & 10,16 \\
\hline Galicia & $-22,28$ & $-62,43$ & 16,44 & 18,84 & $-2,41$ & $-46,00$ & $-109,76$ & 81,70 & 19,31 & 19,99 & $-0,67$ & 101,01 \\
\hline Madrid & $-48,15$ & $-134,95$ & 2,77 & 3,76 & \begin{tabular}{|l|}
$-0,99$ \\
\end{tabular} & $-132,17$ & $-358,98$ & 267,20 & 49,86 & 40,36 & 9,50 & 317,06 \\
\hline Murcia & $-16,25$ & $-45,55$ & $-11,25$ & $-9,37$ & \begin{tabular}{|l|}
$-1,87$ \\
\end{tabular} & $-56,80$ & $-55,05$ & 40,98 & 8,63 & 9,44 & $-0,81$ & 49,60 \\
\hline Navarra & $-5,54$ & $-15,54$ & 1,83 & 2,05 & $-0,22$ & $-13,71$ & $-25,45$ & \begin{tabular}{|l|}
18,94 \\
\end{tabular} & 4,28 & 4,64 & $-0,36$ & 23,22 \\
\hline País Vasco & $-14,06$ & $-39,41$ & 13,80 & 20,81 & \begin{tabular}{|l|}
$-7,00$ \\
\end{tabular} & $-25,61$ & $-97,28$ & 72,41 & 8,85 & 8,58 & 0,27 & 81,26 \\
\hline Rioja (La) & $-2,63$ & $-7,37$ & 3,62 & 4,30 & \begin{tabular}{|l|}
$-0,68$ \\
\end{tabular} & $-3,75$ & $-12,42$ & 9,25 & $-4,25$ & $-4,75$ & 0,50 & 5,00 \\
\hline
\end{tabular}

Fuente: Elaboración propia. 